\title{
IL FLEBILE SUSSURRO DEL CAOS NELL'ARMONIA DEI PIANETI
}

\author{
ANTONIO GIORGILLI $(*)$
}

Sunto. - Si ripercorre lo sviluppo del problema della stabilità del Sistema Solare a partire dall'opera di Keplero. Vengono trattati gli argomenti seguenti: (i) la scoperta da parte dello stesso Keplero della cosiddetta "grande ineguaglianza" di Giove e Saturno; (ii) lo sviluppo della teoria delle perturbazioni a opera di Lagrange e Laplace e il problema delle risonanze; (iii) la scoperta dei moti caotici da parte di Poincaré; (iv) il teorema di Kolmogorov sulla persistenza di moti quasi periodici e la teoria di Nekhoroshev sulla stabilità per tempi esponenzialmente lunghi. Nella parte finale si dà un breve resoconto di alcuni lavori recenti sull'applicabilità dei teoremi di Kolmogorov e Nekhoroshev a modelli realistici del Sistema Solare, mettendo in evidenza il loro ruolo nella discussione del problema della stabilità.

$$
* * *
$$

Abstract. - The hystorical development of the problem of stability of the Solar System is revisited, starting from the work of Kepler. The following topics are included: (i) the discovery of the so called "great inequality" of Jupiter and Saturn by Kepler himself; (ii) the dawn of perturbation theory in the work of Lagrange and Laplace and the problem of resonances; (iii) the discovery of chaotic motions in the work of Poincaré; (iv) the theorem of Kolmogorov on persistence of quasi periodic motions and the theory of Nekhoroshev on stability over exponentially long times. Finally, an account is given concerning some recent work on the actual applicability of the theorems of Kolmogorov and Nekhoroshev to realistic models of the Solar System, thus pointing out their relevance in discussing the problem of stability.

\section{Preludio: L’alba della teoria delle Perturbazioni}

È tradizione fin da tempi remoti considerare il moto dei corpi celesti come la manifestazione di un ordine perfetto. Il filo conduttore, mantenutosi

(*) Istituto Lombardo Accademia di Scienze e Lettere, Milano. Università degli Studi di Milano, Italia. E-mail: antonio.giorgilli@unimi.it 
fino a Copernico e Keplero, può enunciarsi così: "I movimenti dei pianeti sono descrivibili come sourapposizione di periodi (o frequenze). La determinazione dei periodi si fonda sulle osservazioni. "Per i babilonesi si trattava di compilare delle tabelle. I greci hanno utilizzato strumenti geometrici quali cerchi, eccentrici, epicicli ed equanti, grazie ai quali hanno cercato di rappresentare il moto dei pianeti come combinazione di moti circolari uniformi, in pratica l'equivalente dei nostri sviluppi di Fourier.

La scoperta della forma ellittica delle orbite da parte di Keplero può leggersi come una riformulazione sintetica e particolarmente elegante dei modelli classici. In effetti, il moto sull'ellisse potrebbe ben rappresentarsi come combinazione di una infinità di epicicli, di cui però conosciamo la somma in forma chiusa. Gli strumenti geniali ideati dall'astronomia greca vengono consegnati alla storia: cerchi, eccentrici ed epicicli che descrivono l'orbita di un pianeta vengono rimpiazzati da una singola ellisse; gli equanti vengono sostituiti dalla legge delle aree; ciascun pianeta ha un solo periodo.

Di fatto, la notevole semplificazione della geometria delle orbite ha vita breve. Lo stesso Keplero si rende conto che le orbite ellittiche non descrivono in modo del tutto corretto i moti planetari. Credo sia interessante ripercorrere brevemente le tappe di questa scoperta.

Nel 1564 Ticho Brahe, allora diciassettenne, concepisce il progetto di produrre delle tavole astronomiche che sostituiscano le Tavole Alfonsine [1], pubblicate in prima versione ${ }^{1}$ nel 1252 , e ormai obsolete. Al fine di produrre tavole il più possibile precise Ticho Brahe si impegna anche in una campagna di osservazioni e nella costruzione di nuovi strumenti che gli permettono di ridurre sensibilmente gli errori di osservazione. Nel 1572 ha inizio la compilazione delle tavole.

Nel 1600 Ticho Brahe chiama Keplero a Praga, affidandogli il compito di collaborare allo svolgimento di tutti i calcoli necessari, ma senza mettergli a disposizione i dati delle sue osservazioni. Dopo la morte di Brahe, nel 1601, Keplero ha finalmente accesso a quei dati, e grazie a essi imposta il suo lavoro successivo. Nel corso del suo lavoro, ce lo racconta lui stesso nel capitolo VII dell' Astronomia Nova [39], egli si scontra col problema di determinare l'orbita di Marte - che Plinio aveva battezzato "inobservabile sidus". Pur facendo uso del sistema copernicano che pone

1 La voce [1] in bibliografia fa riferimento a una ristampa del 1545, di cui si trova copia nella biblioteca dell'Istituto Lombardo Accademia di Scienze e Lettere, Milano. 
il Sole in prossimità del centro del mondo, il calcolo dell'orbita mediante gli strumenti tradizionali - cerchi, eccentrici, equanti ed epicicli - si rivela praticamente impossibile. Gli innumerevoli sforzi di Keplero sono copiosamente e puntigliosamente documentati nell'Astronomia Nova, dove troviamo l'enunciato delle prime due leggi: la forma dell'orbita è un'ellisse, di cui il Sole occupa un fuoco; la quantità che evolve uniformemente non è un angolo, ma l'area spazzata dal raggio vettore che congiunge il Sole col pianeta.

Nel 1623 Keplero completa la compilazione delle Tabula Rudolphina, così denominate in onore dell'imperatore Rodolfo II, pubblicate nel 1627 [40]. Nella prefazione [41] Keplero così scrive: ${ }^{2}$

" Et de certitudine quidem calculi testabuntur observationes presentium temporum, imprimis Brabeana; de futuris vero temporibus plura prasumere non possumus, quam vel observationes veterum, quibus usus sum, vel ipsa motuum mediorum conditio, nondum penitus explorata, concursusque causarum physicarum prestare possunt, cum observationes Regiomontani et Waltheri testentur, omnino de aquationibus secularibus esse cogitandum, ut singulari libello reddam demonstratum suo tempore; qua tamen aquationes quales et quanta sint, ante plurimum saculorum decursum observationesque eorum, a gente bumana definiri nequaquam possunt.

Il libretto promesso da Keplero non fu pubblicato, ma tra i suoi manoscritti ne venne ritrovata una versione preliminare ${ }^{3}$ che i curatori delle opere hanno incluso nel vol. VI [42]. In quella nota egli confronta i risultati dei suoi calcoli con una serie di osservazioni raccolte da Regiomontano e Walther tra il 1460 e il 1515. Il fatto sorprendente, il solo che Keplero abbia comunicato direttamente ad alcuni corrispondenti, è

2 "E dell'esattezza del calcolo daranno testimonianza le osservazioni del tempo presente, in particolar modo quelle di Brahe; per il futuro invece non possiamo fare troppe previsioni. Le osservazioni del passato, con le quali sono familiare, lo stesso comportamento dei moti medi, il cui studio è ancora incompleto, e il concorso di cause fisiche possono influenzare il calcolo. Infatti le osservazioni di Regiomontano e Walther mettono chiaramente in evidenza la necessità di introdurre dei movimenti secolari: lo mostrerò a suo tempo in un apposito libretto. Quante e quali siano quelle equazioni, l'umanità non potrà saperlo se non dopo molti secoli di osservazioni.” ([41], p. 674.)

3 Nella nota [20], da cui è tratta la Fig.1, si può trovare una sintesi delle argomentazioni di Keplero. 


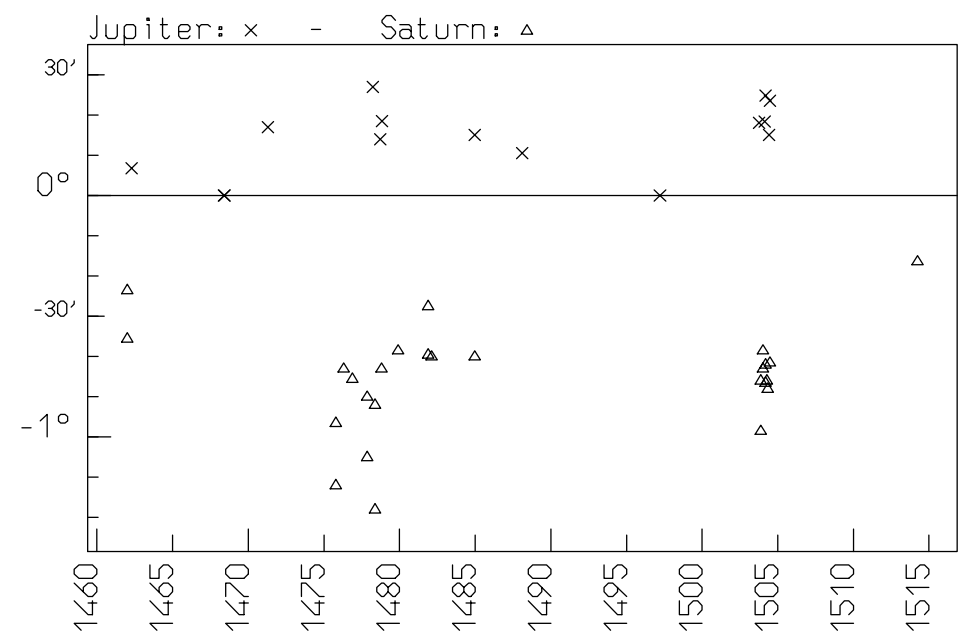

Fig. 1. Differenza tra le longitudini di Giove e Saturno calcolate da Keplero mediante le Tabula Rudolphina e quelle dedotte dalle osservazioni di Regiomontano e Walther, in funzione della data di osservazione.

una deviazione sistematica delle longitudini di Giove e Saturno: Giove sembra accelerare, mentre Saturno sembra rallentare. Il fenomeno è illustrato in Fig. 1, in cui sono riportate le differenze tra le longitudini previste mediante il calcolo e quelle osservate. Pur tenendo conto che gli errori di osservazione sono abbastanza consistenti, trattandosi di valutazioni puramente visuali, la sistematicità delle deviazioni è evidente. Come scrive nella prefazione alle tabulæ, Keplero è convinto della necessità di introdurre delle equazioni secolari, ossia variazioni periodiche molto lente e rivelabili solo sull'arco di secoli, che rendano conto di tali deviazioni. L'impresa si rivela ardua: il calcolo delle frequenze richiede osservazioni distribuite sull'arco, egli dice, di molti secoli, e lascia il compito ai posteri.

Nel 1686 Newton pubblica i Principia [67]. Non credo sia necessario, in questa sede, entrare nei dettagli su come la teoria della gravitazione renda ragione della forma ellittica delle orbite kepleriane - proponendo al tempo stesso una spiegazione dei moti di quegli oggetti misteriosi che sono le comete. Ma vorrei richiamare l'attenzione su due punti.

Il primo punto è il mutamento radicale nel modo di determinare il 
periodo delle orbite, anche nel caso di oggetti che non siano i pianeti. La legge di gravitazione consente di determinare i periodi mediante il calcolo; le osservazioni servono solo per determinare in modo sempre più preciso i parametri delle orbite. Ci si pone dunque la domanda: la gravitazione può giustificare le ineguaglianze messe in evidenza da Keplero?

Il secondo punto riguarda la stabilità del Sistema Solare. Possiamo illustrarlo citando un brano del trattato di Ottica di Newton (1704).

"Now by the help of these Principles, all material Things seem to have been composed of the hard and solid Particles above-mention'd, variously associated in the first Creation by the Counsel of an intelligent Agent. For it became him who created them to set them in order. And if he did so, it's unphilosophical to seek for any other Origin of the World, or to pretend that it might arise out of a Chaos by the mere Laws of Nature; though being once form'd, it may continue by those Laws for many Ages. For while Comets move in very excentrick Orbs in all manner of Positions, blind Fate could never make all the Planets move one and the same way in Orbs concentrick, some inconsiderable Irregularities excepted, which may have risen from the mutual Actions of Comets and Planets upon one another, and which will be apt to increase, till this System wants a Reformation.

In breve, Newton si rende conto perfettamente che il moto ellittico può essere perturbato dall'azione gravitazionale mutua tra i pianeti, e che l'armonia dei moti ellittici potrebbe esserne turbata fino a distruggere, alla lunga, il Sistema Solare. Ma non si azzarda a prevedere quanto tempo occorra per osservare variazioni davvero consistenti, e si limita a invocare l'intervento divino per rimettere le cose a posto, in caso di necessità.

Nel 1719 Halley pubblica delle nuove tavole in cui introduce dei termini secolari. Lo fa in modo del tutto pragmatico: servendosi di dati forniti da Tolomeo (noti anche a Keplero) rinuncia a cercare ineguaglianze periodiche: si limita ad assumere che il moto medio (ossia la velocità angolare media) dei pianeti cambi linearmente nel tempo. In tal modo, afferma, si hanno tavole che possono considerarsi attendibili su un arco temporale di 6000 anni prima e dopo il 1700 . Da notare che Halley usa lo stesso aggettivo secolare introdotto da Keplero, ma rimuove l'idea che si possa trattare di correzioni periodiche.

L'accumulo di osservazioni nella prima metà del secolo XVIII conferma l'esistenza di deviazioni, del resto descritte dalle tavole di Halley, al punto che l'Académie de France bandisce tre premi, negli anni 1748, 1750 
e 1752, aventi per tema lo studio delle ineguaglianze sulla base della teoria newtoniana. I premi del 1748 e del 1752 vengono assegnati a Eulero, che riesce a giustificare le variazioni secolari di Halley (lineari nel tempo), ma con un segno sbagliato: ambedue i pianeti dovrebbero accelerare. Le sue memorie però hanno un merito notevole: in esse si pongono le basi per lo sviluppo di metodi perturbativi. Il premio del 1750 non viene assegnato.

Nei decenni successivi il problema del calcolo delle orbite planetarie tramite la teoria della gravitazione di Newton viene affrontato da Lagrange, presto seguito da un più giovane e arrembante Laplace.

\section{La primavera della Meccanica}

In questo paragrafo vorrei richiamare gli elementi di base della teoria di Lagrange, illustrando in termini più tecnici il problema dei termini secolari e il suo impatto sul problema della stabilità del Sistema Solare. Lo farò ricorrendo alla forma Hamiltoniana delle equazioni, che consente un'esposizione più sintetica. La discussione qui riportata si ispira ampiamente alle Leçons de Mécanique Céleste di Poincaré [71].

\subsection{Gli elementi orbitali}

Al fine di fissare il linguaggio e le notazioni inizierò col richiamare brevemente le definizioni degli elementi orbitali, di uso comune in astronomia, facendo riferimento alle Figg. 2e 3. La comprensione viene facilitata se si pensa di rappresentare l'orbita del pianeta sulla sfera celeste, con centro nel Sole. Scelto un riferimento assoluto con origine nel Sole e assi $x, y, z$, come in Fig.2, si considera un secondo riferimento con assi $\xi, \eta, \zeta$ scelti in modo che $\zeta$ sia ortogonale al piano dell'orbita, a sua volta determinato dall'asse dei nodi $S N$ e dall'asse $\xi$ che congiunge il Sole col perielio dell'orbita del pianeta. L'ellisse viene invece rappresentata direttamente nel piano dell'orbita, in Fig.3. I 6 elementi orbitali formano tre coppie:

$$
\begin{array}{ll}
\iota: \text { inclinazione }, & \Omega: \text { argomento del nodo; } \\
e: \text { eccentricità }, & \omega: \text { argomento del perielio; } \\
a: \text { semiasse maggiore }, & \psi: \text { anomalia vera; }
\end{array}
$$

a questi si aggiungono il periodo $T$, il moto medio $n=\frac{2 \pi}{T}$ e il tempo $\tau$ del passaggio al perielio. Nella descrizione kepleriana la sola quantità dipendente dal tempo è l'anomalia vera $\psi$; le altre sono costanti che devono 


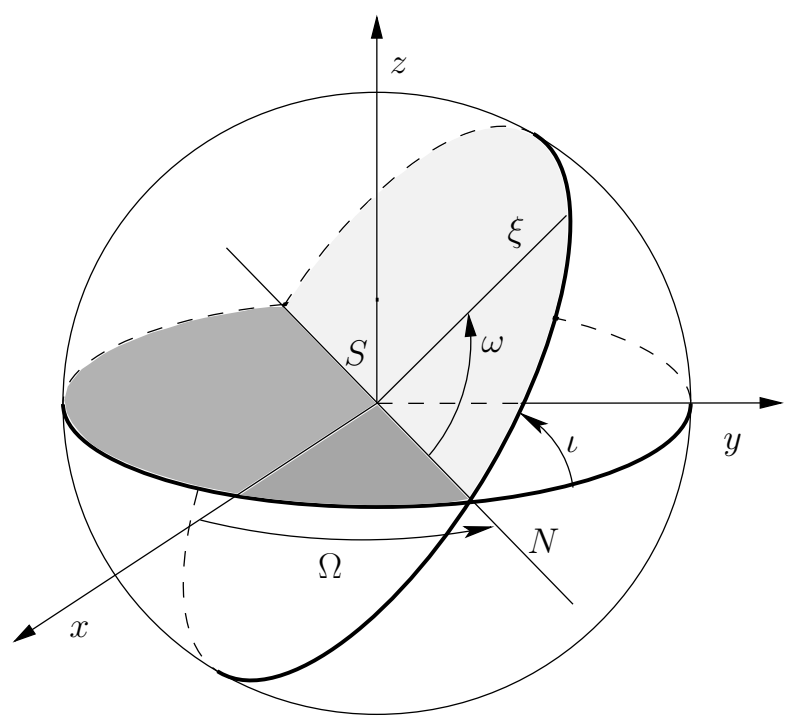

Fig. 2. L'inclinazione $\iota$, l'argomento del nodo $\Omega$ e l'argomento del perielio $\omega$, viste in un riferimento assoluto con origine nel Sole.

essere determinate mediante le osservazioni. In particolare il moto medio $n$ e il semiasse maggiore $a$ soddisfano la relazione $n^{2} a^{3}=4 \pi^{2} K$, dove $K$ è una costante che per la terza legge di Keplero dipende solo dal Sole. Resta da determinare l'anomalia vera $\psi$ come funzione del tempo. L'evoluzione di $\psi(t)$ non è uniforme, ma qui viene in aiuto la seconda legge.

La scrittura di una relazione diretta tra l'area spazzata dal raggio vettore (in grigio in Fig.3) e l'anomalia vera $\psi$ non è affatto agevole. Conviene invece, seguendo la tradizione degli astronomi, introdurre due nuovi angoli: l'anomalia eccentrica $u$ rappresentata in Fig. 3 e l'anomalia media $\ell=n t$. Con qualche considerazione geometrica si ricava la celeberrima equazione di Keplero ${ }^{4}$

$$
u-e \sin u=\ell, \quad \ell=n t,
$$

dove $t$ è il tempo misurato a partire dal passaggio al perielio. La relazione tra $u$ e $\psi$ si ottiene da considerazioni di trigonometria, e può scriversi in

4 L'area del triangolo curvilineo $P S M$ si ricava moltiplicando l'area $P S Q$ per il rapporto $b / a$ tra i semiassi. A sua volta, l'area $P S Q$ si calcola sottraendo al settore circolare $P O Q$ il triangolo $S O Q$. 


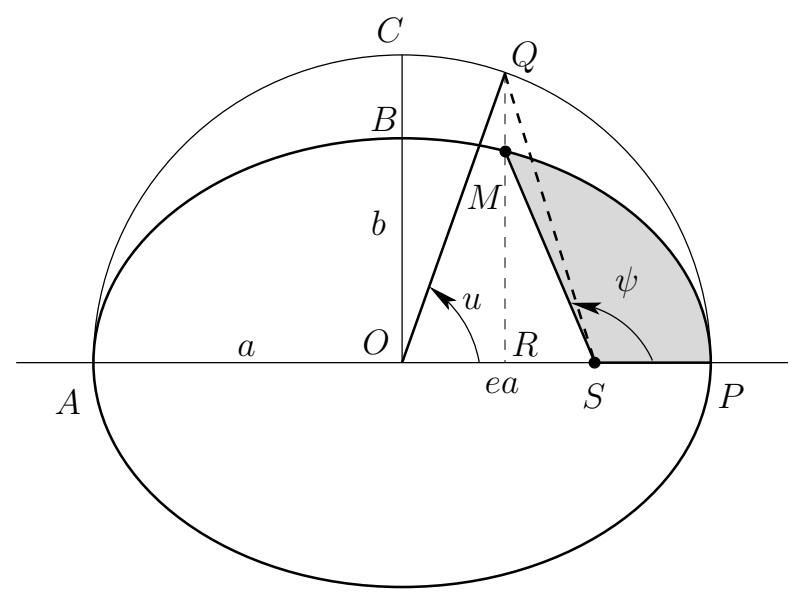

Fig. 3. Gli elementi dell'ellisse: il semiasse maggiore a, l'eccentricità e che misura la distanza ea tra il centro $O$ e il fuoco $S$ ove si trova il Sole, il semiasse minore b. I punti A e P sono rispettivamente l'afelio e il perielio. Detta $M$ la posizione istantanea del pianeta si conduce la perpendicolare al semiasse maggiore e si determinano i punti $R$ e $Q$ intersecando la perpendicolare rispettivamente con l'asse maggiore e con la circonferenza con centro $O$ e raggio a. Si determinano cosi due angoli: l'anomalia eccentrica u e l'anomalia vera $\psi$.

una delle due forme

$$
\tan \frac{u}{2}=\sqrt{\frac{1-e}{1+e}} \tan \frac{\psi}{2}, \quad \sin u=\frac{\sqrt{1-e^{2}} \sin \psi}{1+e \cos \psi} .
$$

\subsection{La formulazione hamiltoniana}

Lagrange sviluppa il suo calcolo facendo uso del metodo della variazione delle costanti, da lui stesso ideato. In altre parole, ammette che le cinque quantità $a, e, \omega, \iota, \Omega$ dipendano dal tempo. La scrittura delle equazioni risulta alquanto laboriosa. Qui farò ricorso al formalismo Hamiltoniano, molto più comodo, ma c'è un prezzo da pagare: gli elementi orbitali non formano un insieme di variabili canoniche. Il procedimento classico consiste nell'introdurre tre coppie di variabili d'angolo-azione, in cui le azioni hanno corrispondenza diretta con i semiassi maggiori, le eccentricità e le inclinazioni. La costruzione di variabili appropriate è dovuta 
a Delaunay [17]. Rimandando altrove per il calcolo, ${ }^{5}$ che comunque richiede un po' di pazienza, riporto solo le relazioni tra queste ultime variabili e gli elementi orbitali.

$$
\begin{aligned}
L_{j} & =\mu_{j} \sqrt{\mathcal{G}\left(m_{0}+m_{j}\right) a}, & & \ell_{j}=n_{j}\left(t-\tau_{j}\right) \\
G_{j} & =L_{j} \sqrt{1-e_{j}^{2}}, & & g_{j}=\omega_{j} \\
\Theta_{j} & =G_{j} \cos \iota_{j}, & \vartheta_{j} & =\Omega_{j}
\end{aligned}
$$

Qui ho denotato con $m_{0}$ la massa del Sole, posto nell'origine, e con $m_{j}$ la massa del $j$-esimo pianeta, e ho fatto uso delle masse ridotte $\mu_{j}=\frac{m_{0} m_{j}}{m_{0}+m_{j}}$.

Riporto in forma alquanto sintetica il procedimento da seguire al fine di scrivere l'Hamiltoniana del sistema planetario in variabili di Delaunay. Seguendo Poincaré [71], farò uso di un sistema di coordinate eliocentriche, in cui lo stato di ciascun pianeta viene descritto mediante le coordinate canoniche $\left(\mathbf{r}_{j}, \mathbf{p}_{j}\right)$, dove $\mathbf{r}_{j}$ è il raggio vettore rispetto al Sole e $\mathbf{p}_{j}$ il momento corrispondente. ${ }^{6}$ In tali variabili l'Hamiltoniana di un sistema di $n$ pianeti più il Sole può spezzarsi in due contributi $H(\mathbf{p}, \mathbf{r})=$ $H_{0}(\mathbf{p}, \mathbf{r})+\varepsilon H_{1}(\mathbf{p}, \mathbf{r})$, dove

$$
H_{0}=\sum_{j=1}^{n}\left(\frac{\mathbf{p}_{j}^{2}}{2 \mu_{j}}-\mathcal{G} \frac{\left(m_{0}+m_{j}\right) \mu_{j}}{\left\|\mathbf{r}_{j}\right\|}\right)
$$

è la parte kepleriana, che descrive il moto intorno al Sole di ciascun pianeta, mentre

$$
\varepsilon H_{1}=\sum_{1 \leq j<k \leq n} \frac{\mathbf{p}_{j} \cdot \mathbf{p}_{k}}{m_{0}}-\mathcal{G} \sum_{1 \leq j<k \leq n} \frac{m_{j} m_{k}}{\left\|\mathbf{r}_{j}-\mathbf{r}_{k}\right\|}
$$

contiene i termini di interazione tra i pianeti. ${ }^{7}$ È uso comune anteporre a

5 Oltre alla memoria di Delaunay, di non facile lettura, si può consultare il trattato di Poincaré [71]. Una deduzione elegante si trova nel trattato di Born [11], ove si fa uso del procedimento classico per la costruzione delle variabili d'azione. Per inciso, l'obiettivo di Born è il calcolo dei livelli quantici dell'atomo di idrogeno, da lui ricavati quantizzando le azioni così costruite.

6 In Meccanica Celeste si fa ampio uso di un altro sistema di coordinate, introdotto da Jacobi, in cui si ordinano i pianeti e si denota con $\mathbf{r}_{j}$ il vettore che identifica la posizione del pianeta rispetto al baricentro dei precedenti. Le considerazioni sul tipo di sviluppi trigonometrici e sulla ricerca di soluzioni riportate in questa nota restano del tutto valide.

7 La scrittura delle equazioni di Newton in coordinate eliocentriche è trattata in modo eccellente in [54], Vol. II, cap. III, $\S 22$. 
$H_{1}$ un fattore $\varepsilon$, pensato come una quantità piccola, al fine di mettere in evidenza il fatto che l'interazione tra i pianeti, a condizione che le orbite restino abbastanza distanziate, è piccola rispetto all'azione del Sole. Ad esempio, si può porre $\varepsilon=\max _{j} m_{j} / m_{0}$, sicché nel caso del sistema solare si ha $\varepsilon \sim 10^{-3}$ (il rapporto tra le masse di Giove e del Sole).

La scrittura dell'Hamiltoniana del sistema planetario in variabili di Delaunay richiede una robusta dose di pazienza e di calcoli che il lettore interessato potrà trovare in molti testi, a partire da quello citato di Poincaré. Qui mi limito alle informazioni rilevanti. La parte Kepleriana dell'Hamiltoniana dipende dalla sola variabile $L$, e assume la forma

$$
H_{0}=-\mathcal{G}^{2} \sum_{j=1}^{n} \frac{\mu_{j}^{3}\left(m_{0}+m_{j}\right)^{2}}{2 L_{j}^{2}},
$$

Osservando che nelle (1) le $L_{j}$ sono in corrispondenza diretta con i semiassi maggiori segue immediatamente che le frequenze dei moti medi (ossia l'evoluzione delle anomalie medie) dipendono solo dal semiasse maggiore. La scrittura della perturbazione $H_{1}(L, G, \Theta, \ell, g, \vartheta)$ in forma utile è faccenda decisamente più laboriosa. Avendo espresso le coordinate $\mathbf{r}_{j} \mathrm{e}$ i momenti $\mathbf{p}_{j}$ in funzione delle variabili di Delaunay occorre sviluppare $H_{1}$ in serie trigonometrica degli angoli $\ell, g, \vartheta$. Ai fini della discussione basta dire che si ottengono delle serie trigonometriche con coefficienti dipendenti da $L, G, \Theta$. Ma le difficoltà non sono finite, perché le variabili di Delaunay hanno almeno due difetti. Il primo è che per $e=0$, ossia per le orbite circolari, le variabili $L$ e $G$ coincidono, e l'argomento del perielio risulta indefinito. La seconda è che per $\iota=0$, ossia per le orbite a inclinazione nulla, le variabili $G$ e $\Theta$ coincidono, e risulta indefinito l'argomento del nodo. Ora, i metodi perturbativi diventano efficaci proprio quando l'eccentricità $e$ e l'inclinazione $\iota$ sono prossime a zero, e questo è proprio il caso del Sistema Solare. Per superare le difficoltà si preferisce introdurre il nuovo sistema delle variabili di Delaunay modificate, definite mediante la trasformazione canonica lineare

$$
\begin{aligned}
& \Lambda_{j}=L_{j} \quad, \quad \lambda_{j}=\ell_{j}+g_{j}+\vartheta_{j} \quad \text { (longitudine media) } \\
& P_{j}=L_{j}-G_{j}, \quad p_{j}=-g_{j}-\vartheta_{j} \quad \text { (longitudine del perielio) } \\
& Q_{j}=G_{j}-\Theta_{j}, \quad q_{j}=-\vartheta_{j} \quad \text { (longitudine del nodo) }
\end{aligned}
$$

A ben vedere il problema della singolarità per eccentricità e inclinazione nulle non viene risolto completamente, perché per $P_{j}=0$ o $Q_{j}=0$ gli angoli corrispondenti $p_{j}$ e $q_{j}$ risultano ancora indefiniti. È però interessante 
il fatto che in queste variabili la perturbazione assume la forma particolare di uno sviluppo in serie trigonometrica degli angoli con coefficienti che sono polinomi in $P_{j}^{1 / 2}$ e $Q_{j}^{1 / 2}$, che può scriversi genericamente come

$$
\begin{array}{r}
H_{1}=\sum_{\boldsymbol{\alpha}, \boldsymbol{\beta} \in \mathbb{Z}_{+}^{n}} \sum_{\mathbf{k}, \mathbf{m}, \mathbf{s} \in \mathbb{Z}^{n}} c_{\boldsymbol{\alpha}, \boldsymbol{\beta}, \mathbf{k}, \mathbf{m}, \mathbf{s}}(\boldsymbol{\Lambda}) \mathbf{P}^{\boldsymbol{\alpha} / 2} \mathbf{Q}^{\boldsymbol{\beta} / 2} \times \\
\exp (i\langle\mathbf{k}, \boldsymbol{\lambda}\rangle+i\langle\mathbf{m}, \mathbf{p}\rangle+i\langle\mathbf{s}, \mathbf{q}\rangle),
\end{array}
$$

dove restano da determinare i coefficienti $c_{\boldsymbol{a}, \boldsymbol{\beta}, \mathbf{k}, \mathbf{m}, \mathbf{s}}(\boldsymbol{\Lambda})$. (Qui, ho fatto uso di una notazione di tipo vettoriale per $\boldsymbol{\Lambda}=\left(\Lambda_{1}, \ldots, \Lambda_{N}\right)$ e per tutte le altre variabili.) Ma non è tutto. Il fatto ancor più interessante è che non tutte le combinazioni $\langle\mathbf{k}, \boldsymbol{\lambda}\rangle+\langle\mathbf{m}, \mathbf{p}\rangle+\langle\mathbf{s}, \mathbf{q}\rangle$ compaiono effettivamente nello sviluppo: ci sono delle restrizioni molto forti, dette regole $d i$ D'Alembert, su cui non mi soffermo in dettaglio, ma mi limito a mettere in evidenza la conseguenza davvero rilevante. La trasformazione canonica alle variabili di Poincaré $\xi, \eta$

$$
\begin{aligned}
\xi_{j} & =\sqrt{2 P_{j}} \cos p_{j}, & \eta_{j} & =\sqrt{2 P_{j}} \sin p_{j}, \\
\xi_{j+n} & =\sqrt{2 Q_{j}} \cos q_{j}, & \eta_{j+n} & =\sqrt{2 Q_{j}} \sin q_{j}
\end{aligned}
$$

cambia l'Hamiltoniana in $H(\boldsymbol{\Lambda}, \boldsymbol{\lambda}, \boldsymbol{\xi}, \boldsymbol{\eta})=H_{0}(\boldsymbol{\Lambda})+\varepsilon H_{1}(\boldsymbol{\Lambda}, \boldsymbol{\lambda}, \boldsymbol{\xi}, \boldsymbol{\eta})$, dove

$$
\begin{aligned}
H_{0} & =-\mathcal{G}^{2} \sum_{j=1}^{n} \frac{\mu_{j}^{3}\left(m_{0}+m_{j}\right)^{2}}{2 \Lambda_{j}^{2}}, \\
H_{1} & =\sum_{\boldsymbol{\alpha}, \boldsymbol{\beta} \in \mathbb{N}_{0}^{2 n}} \sum_{\mathbf{k} \in \mathbb{Z}^{n}} c_{\boldsymbol{\alpha}, \boldsymbol{\beta}, \mathbf{k}}(\boldsymbol{\Lambda}) \xi^{\boldsymbol{\alpha}} \boldsymbol{\eta}^{\boldsymbol{\beta}} \exp (i\langle\mathbf{k}, \boldsymbol{\lambda}\rangle),
\end{aligned}
$$

con dei coefficienti $c_{\boldsymbol{\alpha}, \boldsymbol{\beta}, \mathbf{k}}(\boldsymbol{\Lambda})$ che possono essere determinati. Qui ho denotato con $\mathbb{N}_{0}$ l'insieme dei numeri interi non negativi. Il fatto realmente sorprendente e inaspettato è la dipendenza puramente polinomiale dalle variabili $\xi, \eta$ che descrivono le eccentricità e le inclinazioni, e che si comportano come coordinate cartesiane nell'intorno dell'origine. $\mathrm{A}$ dire il vero c'è qualcosa di ancor più sorprendente, ma lo rimando al paragrafo 2.4, perché quanto ho detto fin qui basta per illustrare il risultato di Lagrange sulla stabilità del Sistema Solare. 


\subsection{L'invarianza dei semiassi maggiori}

Le equazioni canoniche per l'Hamiltoniana (6) sono

$$
\begin{aligned}
& \dot{\lambda}_{j}=\frac{\mu_{j}^{3}\left(m_{0}+m_{j}\right)^{2}}{\Lambda_{j}^{3}} \\
& \quad+\varepsilon \sum_{\boldsymbol{a}, \boldsymbol{\beta} \in \mathbb{N}_{0}^{2 n}} \sum_{\mathbf{k} \in \mathbb{Z}^{n}} \frac{\partial c_{\boldsymbol{\alpha}, \boldsymbol{\beta}, \mathbf{k}}}{\partial \Lambda_{j}}(\boldsymbol{\Lambda}) \xi^{\boldsymbol{a}} \boldsymbol{\eta}^{\boldsymbol{\beta}} \exp (i\langle\mathbf{k}, \boldsymbol{\lambda}\rangle), \\
& \dot{\Lambda}_{j}=\quad-\varepsilon \sum_{\boldsymbol{\alpha}, \boldsymbol{\beta} \in \mathbb{N}_{0}^{2 n}} \sum_{\mathbf{0} \neq \mathbf{k} \in \mathbb{Z}^{n}} i k_{j} c_{\boldsymbol{\alpha}, \boldsymbol{\beta}, \mathbf{k}}(\boldsymbol{\Lambda}) \xi^{\alpha} \boldsymbol{\eta}^{\boldsymbol{\beta}} \exp (i\langle\mathbf{k}, \boldsymbol{\lambda}\rangle), \\
& \dot{\xi}_{j}=\quad \varepsilon \sum_{\boldsymbol{\alpha}, \boldsymbol{\beta} \in \mathbb{N}_{0}^{2 n}} \sum_{\mathbf{k} \in \mathbb{Z}^{n}} \frac{\beta_{j}}{\eta_{j}} c_{\boldsymbol{\alpha}, \boldsymbol{\beta}, \mathbf{k}}(\boldsymbol{\Lambda}) \xi^{\boldsymbol{\alpha}} \boldsymbol{\eta}^{\boldsymbol{\beta}} \exp (i\langle\mathbf{k}, \boldsymbol{\lambda}\rangle), \\
& \dot{\eta}_{j}=\quad-\varepsilon \sum_{\boldsymbol{\alpha}, \boldsymbol{\beta} \in \mathbb{N}_{0}^{2 n}} \sum_{\mathbf{k} \in \mathbb{Z}^{n}} \frac{\alpha_{j}}{\xi_{j}} c_{\boldsymbol{\alpha}, \boldsymbol{\beta}, \mathbf{k}}(\boldsymbol{\Lambda}) \xi^{\alpha} \boldsymbol{\eta}^{\boldsymbol{\beta}} \exp (i\langle\mathbf{k}, \boldsymbol{\lambda}\rangle),
\end{aligned}
$$

Alle equazioni appena trovate si applica un procedimento di soluzione per approssimazioni successive. Si cercano le soluzioni sotto forma di serie di potenze in $\varepsilon$, ossia

$$
\begin{aligned}
\lambda_{j} & =\lambda_{j, 0}(t)+\varepsilon \lambda_{j, 1}(t)+\ldots, & \Lambda_{j} & =\Lambda_{j, 0}(t)+\varepsilon \Lambda_{j, 1}(t)+\ldots, \\
\xi_{j} & =\xi_{j, 0}(t)+\varepsilon \xi_{j, 1}(t)+\ldots, & \eta_{j} & =\eta_{j, 0}(t)+\varepsilon \eta_{j, 1}(t)+\ldots,
\end{aligned}
$$

dove $\lambda_{j, s}(t), \Lambda_{j, s}(t), \xi_{j, s}(t), \eta_{j, s}(t)$ sono funzioni da determinarsi. Sostituendo queste espressioni in ambo i membri e sviluppando in serie di $\varepsilon$ si costruisce la soluzione per confronto dei coefficienti, ossia eguagliando separatamente i termini dello stesso grado in $\varepsilon$.

All'ordine zero, isolando il coefficiente di $\varepsilon^{0}$, si hanno le equazioni

$$
\dot{\lambda}_{j, 0}=\frac{\mu_{j}^{3}\left(m_{0}+m_{j}\right)^{2}}{\Lambda_{j, 0}^{3}}, \quad \dot{\Lambda}_{j, 0}=0, \quad \dot{\xi}_{j, 0}=0, \quad \dot{\eta}_{j, 0}=0 .
$$

La facile soluzione di queste equazioni ci dà la descrizione kepleriana delle orbite, con $\Lambda_{j, 0}, \varphi_{j, 0}, \xi_{j, 0}, \eta_{j, 0}$ costanti e

$$
\lambda_{j, 0}(t)=\nu_{j} t+\varphi_{j, 0}, \quad \nu_{j}=\frac{\mu_{j}^{3}\left(m_{0}+m_{j}\right)^{2}}{\Lambda_{j, 0}^{3}} .
$$

Sostituendo questa soluzione nei secondi membri delle equazioni (7) si 
ottengono le equazioni per l'ordine $\varepsilon$, che si scrivono

$$
\begin{aligned}
& \dot{\lambda}_{j, 1}=-\frac{3 \mu_{j}^{3}\left(m_{0}+m_{j}\right)^{2}}{\Lambda_{j, 0}^{4}} \Lambda_{j, 1} \\
& +\sum_{\boldsymbol{\alpha}, \boldsymbol{\beta} \in \mathbb{N}_{0}^{2 n}} \sum_{\mathbf{k} \in \mathbb{Z}^{n}} \frac{\partial c_{\boldsymbol{\alpha}, \boldsymbol{\beta}, \mathbf{k}}}{\partial \Lambda_{j}}\left(\boldsymbol{\Lambda}_{0}\right) \xi_{0}^{\boldsymbol{\alpha}} \boldsymbol{\eta}_{0}^{\boldsymbol{\beta}} \exp \left(i\left\langle\mathbf{k}, \boldsymbol{\lambda}_{0}\right\rangle\right), \\
& \dot{\Lambda}_{j, 1}=-\sum_{\boldsymbol{\alpha}, \boldsymbol{\beta} \in \mathbb{N}_{0}^{2 n}} \sum_{\mathbf{0} \neq \mathbf{k} \in \mathbb{Z}^{n}} i k_{j} c_{\boldsymbol{\alpha}, \boldsymbol{\beta}, \mathbf{k}}\left(\boldsymbol{\Lambda}_{0}\right) \xi_{0}^{\alpha} \eta_{0}^{\boldsymbol{\beta}} \exp \left(i\left\langle\mathbf{k}, \boldsymbol{\lambda}_{0}\right\rangle\right), \\
& \dot{\xi}_{j, 1}=\sum_{\boldsymbol{\alpha}, \boldsymbol{\beta} \in \mathbb{N}_{0}^{2 n}} \sum_{\mathbf{k} \in \mathbb{Z}^{n}} \frac{\beta_{j}}{\eta_{j, 0}} c_{\boldsymbol{\alpha}, \boldsymbol{\beta}, \mathbf{k}}\left(\boldsymbol{\Lambda}_{0}\right) \xi_{0}^{\boldsymbol{\alpha}} \boldsymbol{\eta}_{0}^{\boldsymbol{\beta}} \exp \left(i\left\langle\mathbf{k}, \boldsymbol{\lambda}_{0}\right\rangle\right), \\
& \dot{\eta}_{j, 1}=-\sum_{\boldsymbol{\alpha}, \boldsymbol{\beta} \in \mathbb{N}_{0}^{2 n}} \sum_{\mathbf{k} \in \mathbb{Z}^{n}} \frac{\alpha_{j}}{\xi_{j, 0}} c_{\boldsymbol{a}, \boldsymbol{\beta}, \mathbf{k}}\left(\boldsymbol{\Lambda}_{0}\right) \xi_{0}^{\boldsymbol{\alpha}} \boldsymbol{\eta}_{0}^{\boldsymbol{\beta}} \exp \left(i\left\langle\mathbf{k}, \boldsymbol{\lambda}_{0}\right\rangle\right),
\end{aligned}
$$

dove occorre sostituire la soluzione kepleriana $\boldsymbol{\lambda}_{0}(t)$ appena trovata, mentre le altre quantità $\Lambda_{0}, \xi_{0}, \eta_{0}$ sono costanti. L'aspetto un po' terrificante di queste equazioni non deve spaventare: sono tutte equazioni lineari non omogenee con termine noto trigonometrico, della forma

$$
\dot{x}=\sum_{\mathbf{k} \in \mathbb{Z}^{n}} d_{\mathbf{k}} \exp (i\langle\mathbf{k}, \boldsymbol{v}\rangle t),
$$

la cui soluzione formale si scrive immediatamente, avendosi

$$
x(t)=d_{0} t+\sum_{\mathbf{k} \neq 0} \frac{d_{\mathbf{k}}}{i\langle\mathbf{k}, \boldsymbol{v}\rangle} \exp (i\langle\mathbf{k}, \boldsymbol{v}\rangle t) .
$$

Qui occorre un po' di attenzione: qualcuno dei denominatori $\langle\mathbf{k}, \boldsymbol{v}\rangle$ potrebbe annullarsi, nel qual caso il termine corrispondente dovrebbe riscriversi con una dipendenza lineare dal tempo: si tratta di una risonanza. A tale problema si rimedia semplicemente assumendo che ciò non si verifichi, imponendo la condizione di non risonanza $\langle\mathbf{k}, \boldsymbol{v}\rangle \neq 0$. Nel calcolo esplicito per il caso del nostro Sistema Solare si dovrà necessariamente ricorrere a un troncamento delle serie, tenendo conto di un numero finito di termini trigonometrici. Basta imporre la condizione di non risonanza solo per un numero finito di casi, che risulta soddisfatta.

Resta un secondo problema: il termine di media del secondo membro, qui sopra denotato con $d_{0}$, introduce un termine $d_{0} t$ nella soluzione. Questo sarebbe proprio un termine secolare del tipo introdotto da Halley: una quantità che evolve linearmente nel tempo. Ma se si guarda bene 
la seconda delle equazioni (11) si vede che il termine di media è assente, e di conseguenza la prima correzione $\boldsymbol{\Lambda}_{1}(t)$ dipende dal tempo solo in forma trigonometrica: non ci sono termini secolari (nel senso di Halley). Il motivo è molto semplice: l'equazione per $\Lambda_{j}$ si calcola derivando l'Hamiltoniana rispetto all'angolo $\lambda_{j}$, e dunque il termine noto non può contenere un termine di media, che viene annullata dalla derivata. $\mathrm{Ci}$ potrebbero essere invece termini secolari nelle soluzioni per $\xi_{1}(t)$ e $\boldsymbol{\eta}_{1}(t)$, ma a questi si penserà dopo.

Lagrange si ferma a questo punto, accontentandosi dell'approssimazione del primo ordine nelle masse, o di ordine $\varepsilon$. Il risultato spettacolare di Lagrange sulla stabilità del Sistema Solare si riassume così: Se le frequenze dei moti medi dei pianeti non sono soggette a risonanza, allora l'evoluzione dei semiassi maggiori delle orbite planetarie, calcolata nell'approssimazione del primo ordine nelle masse, non contiene termini secolari.

Prima di proseguire con la teoria di Lagrange vorrei tornare un momento sul problema dell'ineguaglianza di Giove e Saturno. Con la memoria che ho riassunto Lagrange pone un punto fermo, ma allo stesso tempo dimostra l'assenza dei termini secolari di Halley. Il problema delle ineguaglianze di Giove e Saturno viene dunque riaperto. L'enigma viene sciolto nel 1785 da Laplace. L'osservazione cruciale è che qualcuno dei denominatori $\langle\mathbf{k}, \boldsymbol{v}\rangle$ che compaiono nelle soluzioni delle equazioni (11), e in particolare dell'equazione per $\boldsymbol{\Lambda}_{1}$, potrebbe essere molto piccolo, pur non annullandosi. In tal caso il coefficiente corrispondente potrebbe diventare grande, generando una variazione periodica consistente. L'argomento si applica perfettamente al caso di Giove e Saturno, perché le frequenze dei loro moti medi hanno un rapporto prossimo a $5 / 2$. Laplace svolge il calcolo isolando il termine risonante corrispondente e trova una variazione periodica apprezzabile dei semiassi maggiori dei due pianeti, con un periodo di circa 900 anni. Il calcolo rende conto delle variazioni osservate: un grande successo che conferma la validità della teoria di Newton.

\subsection{Le precessioni dei perieli e dei nodi}

Risolto elegantemente il problema dell'evoluzione dei semiassi maggiori (sia pure, osserveremmo noi, in modo approssimato) Lagrange prende in considerazione le inclinazioni e le longitudini dei nodi [45]. Pochi mesi dopo Laplace, ${ }^{8}$ avendo letto il manoscritto di Lagrange, estende la teoria

8 I riferimenti bibliografici possono sollevare qualche perplessità, perché la memoria 
tenendo conto anche delle eccentricità e dei perieli [48].

Anche qui riporto schematicamente il procedimento, rimandando per i dettagli del calcolo al trattato di Poincaré [71]. Lagrange introduce quello che in Meccanica Celeste viene solitamente chiamato modello secolare. Precisamente, partendo dall'Hamiltoniana (6), egli applica il metodo della media approssimando $H_{1}$ con la sua media sugli angoli veloci $\lambda$, tenendo così fissato il valore di $\boldsymbol{\Lambda}$. In pratica, l'Hamiltoniana $H_{0}(\boldsymbol{\Lambda})$ diventa una costante che si può rimuovere, e nello sviluppo trigonometrico di $H_{1}(\boldsymbol{\Lambda}, \boldsymbol{\lambda}, \xi, \boldsymbol{\eta})$ si rimuovono tutti i termini dipendenti da $\boldsymbol{\lambda}$ conservando il solo termine $\mathbf{k}=0$ della somma su $\mathbf{k}$. Si ottiene così la cosiddetta Hamiltoniana secolare

$$
H_{\mathrm{sec}}(\boldsymbol{\xi}, \boldsymbol{\eta})=\sum_{\boldsymbol{\alpha}, \boldsymbol{\beta} \in \mathbb{N}_{0}^{2 n}} c_{\boldsymbol{\alpha}, \boldsymbol{\beta}, \mathbf{0}}\left(\boldsymbol{\Lambda}_{0}\right) \xi^{\alpha} \boldsymbol{\eta}^{\boldsymbol{\beta}}
$$

in cui i coefficienti $c_{\mathbf{\alpha}, \boldsymbol{\beta}, \mathbf{0}}\left(\boldsymbol{\Lambda}_{0}\right)$ diventano costanti. Qui bisogna tornare un momento alla definizione (5) delle variabili di Poincaré. Si nota subito che le prime $n$ componenti dei vettori $\xi, \eta$ descrivono le eccentricità e i perieli, mentre le restanti $n$ descrivono le inclinazioni e i nodi. Fin qui è facile, ma ora viene la parte curiosa, ai limiti del miracolo, che segue dalle regole di D'Alembert. Si hanno due proprietà notevoli.

(i) L'Hamiltoniana secolare può svilupparsi in serie di potenze della forma

$$
H_{\mathrm{sec}}(\boldsymbol{\xi}, \boldsymbol{\eta})=H_{2}(\boldsymbol{\xi}, \boldsymbol{\eta})+H_{4}(\boldsymbol{\xi}, \boldsymbol{\eta})+H_{6}(\boldsymbol{\xi}, \boldsymbol{\eta})+\ldots
$$

in cui compaiono solo termini di grado pari.

(ii) La parte quadratica $H_{2}(\boldsymbol{\xi}, \boldsymbol{\eta})$ si spezza in due parti indipendenti

$$
\begin{aligned}
& H_{2}(\boldsymbol{\xi}, \boldsymbol{\eta})=H_{\text {ecc }}\left(\xi_{1}, \ldots, \xi_{n}, \eta_{1}, \ldots, \eta_{n}\right)+ \\
& H_{\text {obl }}\left(\xi_{n+1}, \ldots, \xi_{2 n}, \eta_{n+1}, \ldots, \eta_{2 n}\right),
\end{aligned}
$$

dove $H_{\text {ecc }}$ dipende solo dalle variabili che descrivono le eccentricità e le longitudini dei perieli, e $H_{\text {obl }}$ dipende solo dalle variabili che descrivono le inclinazioni e le longitudini dei nodi (l'etichetta "obl" sta per "obliquità").

L'Hamiltoniana quadratica descrive di fatto l'evoluzione delle cosiddette variabili lente, ossia eccentricità, inclinazioni, perieli e nodi, riducendola

di Lagrange è stata pubblicata tre anni dopo quella di Laplace. Lo stesso Laplace però riconosce che la sua memoria è un'estensione di quella di Lagrange, di cui era a conoscenza. 
allo studio delle piccole oscillazioni intorno a un equilibrio rappresentato dalle orbite circolari a inclinazione nulla.

A questo punto Lagrange risolve esplicitamente le equazioni per l'approssimazione quadratica $H_{\text {obl }}$ dell'Hamiltoniana che descrive inclinazioni e nodi. Trattandosi di equazioni lineari a coefficienti costanti, egli non fa altro che applicare il metodo da lui stesso ideato [44]. Laplace, a sua volta, non deve far altro che estendere lo stesso metodo alla parte $H_{\text {ecc }}$ che descrive eccentricità e perieli. Il calcolo non è banale, perché si tratta - nel nostro linguaggio - di calcolare numericamente autovalori e autovettori di matrici $6 \times 6$, tenendo conto dei sei pianeti noti a quel tempo (la scoperta di Urano è del 1781), ma è un poco facilitato dal fatto che le matrici hanno diagonali dominanti.

Servendosi delle soluzioni così calcolate Lagrange valuta la variazione massima delle inclinazioni e delle eccentricità, trovandola abbastanza piccola da poter considerare l'approssimazione delle piccole oscillazioni come attendibile. Ne conclude che le eccentricità e le inclinazioni compiono oscillazioni che restano limitate per tutti i tempi, mentre gli angoli sono soggetti a moti di precessione dei nodi e dei perieli dei pianeti, con periodi dell'ordine delle migliaia di anni.

Fin qui la teoria di Lagrange. Se si accettano come ragionevoli tutte le approssimazioni introdotte la si può considerare come una risposta soddisfacente al problema della stabilità del Sistema Solare. Ma in termini rigorosi, e senza nulla togliere ai grandissimi meriti di Lagrange, non la si può considerare definitiva. Restano almeno due punti di chiarire. Il primo è il superamento dell'approssimazione nelle masse; ne parlo nel prossimo paragrafo. Il secondo è la giustificazione dell'approssimazione lineare nello studio dei moti secolari. La non linearità gioca un ruolo essenziale, perché può modificare in modo sostanziale l'evoluzione di nodi e inclinazioni, fino a contraddire le valutazioni di Lagrange sui valori massimi. La vicenda non si chiude qui.

\subsection{Il problema dei termini secolari}

I risultati di Lagrange sollevano una domanda che attraversa tutto il secolo XIX: si può mostrare che l'assenza di termini secolari nell'evoluzione dei semiassi maggiori vale a qualunque ordine nelle masse? Si può porre la questione in termini più precisi ricorrendo alla classificazione di Poincaré. Si distinguono tre tipi di termini:

a. trigonometrici puri, del tipo $\exp (i\langle\mathbf{k}, \boldsymbol{v}\rangle t)$, in cui il tempo appare sotto il segno di una funzione trigonometrica; 
b. secolari misti, del tipo $t^{s} \exp (i\langle\mathbf{k}, \boldsymbol{v}\rangle t)$, prodotto di un polinomio con una funzione trigonometrica in $t$;

c. secolari puri, del tipo $t^{s}$, puramente polinomiali nel tempo.

La teoria di Lagrange afferma che al primo ordine nelle masse l'evoluzione dei semiassi maggiori contiene solo termini trigonometrici puri. D'altra parte non si può escludere che agli ordini successivi compaiano termini secolari misti o secolari puri, che in tal caso si propagherebbero generando potenze di $t$ sempre più elevate. Questo si vede studiando le soluzioni di un'equazione della forma $\dot{x}=t^{s} \exp (i\langle\mathbf{k}, \boldsymbol{v}\rangle t)$ oppure $\dot{x}=t^{s}$ con una potenza $s \geq 0$. La domanda che si pone è: possiamo garantire che a qualunque ordine nelle masse l'evoluzione dei semiassi maggiori non contiene termini secolari puri?

Le speranze cominciano a vacillare quando, nel 1809, Poisson scopre che la soluzione al secondo ordine contiene dei termini secolari misti della forma $t \exp (i\langle\mathbf{k}, \boldsymbol{v}\rangle t)$, ossia prodotti di termini lineari in $t$ con termini trigonometrici. Non ci sono però termini secolari puri. Ciò induce Poisson a riformulare il problema della stabilità del Sistema Solare nel modo seguente: Possiamo dimostrare che il sistema tornerà infinite volte vicino al suo stato iniziale? L'esistenza di termini secolari misti non esclude questa possibilità, e il problema si sposta un poco più in là: dimostrare che non ci sono termini secolari puri. L'illusione cade definitivamente nel 1878, quando Spiru Haretu, uno studente rumeno che sta preparando la sua tesi di dottorato a Parigi, scopre che al terzo ordine nelle masse ci sono dei termini secolari puri [34][35].

Nel frattempo però i matematici e gli astronomi non sono stati a guardare: hanno cercato e trovato dei metodi perturbativi che non generano termini secolari. Sembra una faccenda misteriosa, ma alla base c'è un'osservazione già fatta da Lagrange [44].

Se si applicano i metodi perturbativi che ho descritto all'equazione di un oscillatore non lineare, ad esempio $\ddot{x}+\omega^{2} x=\varepsilon x^{3}$, si vede rapidamente che vengono generati termini secolari sia misti che puri. Ma l'equazione è integrabile, dato che ammette l'integrale primo dell'energia, e le soluzioni, almeno per piccola ampiezza, sono tutte periodiche, e dunque dovrebbero scriversi come serie di Fourier. Come si spiegano i termini secolari? Il fatto è che nel procedimento perturbativo si impone, di fatto, che la frequenza del movimento sia sempre $\omega$, quella del sistema imperturbato. Ma l'equazione è non lineare, e la frequenza vera differisce da $\omega$ di una quantità dipendente dall'ampiezza. Supponendo che la frequenza sia $\omega+\delta$, si può svolgere un rapido calcolo che comprende uno 
sviluppo in serie:

$$
\begin{aligned}
& \sin ((\omega+\delta) t)=\sin \omega t \cos \delta t+\cos \omega t \sin \delta t \\
& \quad=\left(1-\frac{\delta^{2} t^{2}}{2 !}+\frac{\delta^{4} t^{4}}{4 !}+\ldots\right) \sin \omega t+\left(\frac{\delta t}{1 !}-\frac{\delta^{3} t^{3}}{3 !}+\ldots\right) \cos \omega t .
\end{aligned}
$$

La soluzione perturbativa produce, passo passo, gli sviluppi in serie dell'ultima espressione. Un'osservazione che sembra elementare, ma come modificare il procedimento perturbativo in modo da tenerne conto?

Per dare una risposta soddisfacente all'ultima domanda si deve aspettare la seconda metà del secolo XIX, ad opera di Lindstedt [56] e Gyldèn [33]. Il procedimento più noto è quello di Lindstedt. Egli studia il caso di un equilibrio ellittico (o, nel linguaggio abituale in Fisica, un sistema di oscillatori non lineari). Se ne può trovare una breve esposizione in [22]. Considerando un'equazione della forma

$$
\ddot{x}+\omega^{2} x=\Psi_{0}(t)+\Psi_{1}(t) x+\Psi_{2}(t) x^{2}+\ldots
$$

dove $\Psi_{1}(t), \Psi_{1}(t), \Psi_{2}(t), \ldots$ sono funzioni periodiche del tempo con periodo $2 \pi / \lambda$ con $\lambda \neq \omega$ egli modifica il procedimento perturbativo di Lagrange cercando soluzioni di frequenza $\omega+\delta(a)$ dove $a$ è l'ampiezza dell'oscillazione e $\delta(a)$ una funzione dell'ampiezza da determinarsi. Lo stesso procedimento si applica a sistemi di equazioni dello stesso tipo o anche, come mostrato da Poincaré ([70] tomo II, cap. IX) a sistemi più generali.

Il fatto interessante è che, a patto di assumere la non risonanza, si possono costruire formalmente soluzioni che contengono solo termini trigonometrici puri. Si potrebbe parlare di rivincita degli epicicli.

\section{L'URAGANO}

Il problema dei termini secolari e della costruzione di metodi perturbativi atti a evitarli occupa una parte consistente del lavoro dei matematici del secolo XIX. Nel frattempo, dopo la formalizzazione del concetto di convergenza delle serie a opera di Cauchy, si pone la domanda se le serie costruite mediante i metodi perturbativi siano o no convergenti. Questo stato di cose è ben rappresentato dal tema n. 1 del concorso bandito in occasione del $60^{\text {mo }}$ compleanno del Re di Svezia Oscar II, come è stato pubblicato su Nature il 30.7.1885. 
"A system being given of a number whatever of particles attracting one another mutually according to Newton's law, it is proposed, on the assumption that there never takes place an impact of two particles to expand the coordinates of each particle in a series proceeding according to some known function of time and converging uniformly for any space of time.

It seems that this problem, the solution of which will considerably enlarge our knowledge regarding the system of the Universe, might be solved by means of the analytical resources at our present disposition; this may at least be fairly supposed, because shortly before bis death Lejeune-Dirichlet communicated to a friend of his, a mathematician, that he had discovered a method of integrating the differential equations of mechanics, and he had succeeded, by applying this method, to demonstrate the stability of our planetary system in an absolutely strict manner. Unfortunately we know nothing about this method except that the starting point for his discovery seems to have been the theory of infinitely small oscillations. It may, however, be supposed almost with certainty that this method was not based on long and complicated calculations but on the development of a simple fundamental idea, which we may reasonably hope to find again by means of earnest and persevering study.

La scelta di proporre questo tema è probabilmente dovuta a Weierstrass. Egli aveva ideato un metodo per scrivere le soluzioni del problema planetario in forma di serie puramente trigonometriche, ma non era in grado di dimostrarne la convergenza.

Il premio venne assegnato a Poincaré, ma la vicenda non fu del tutto indolore. Strettamente parlando, la memoria di Poincaré non risponde al quesito posto: dimostra semmai il contrario. Inoltre la memoria pubblicata [69] non è quella presentata per il premio, ma una versione ampiamente rielaborata in cui Poincaré corregge un errore presente nella prima, e scopre l'esistenza di moti caotici. ${ }^{9}$ Un errore molto fecondo! Nel paragrafo 3.2 cercherò di descrivere con qualche dettaglio il fenomeno del caos scoperto da Poincaré.

\subsection{Integrabilità e non integrabilità}

Il contenuto della memoria appena menzionata venne ripreso e ampliato considerevolmente da Poincaré nel trattato Métodes Nouvelles. Nel

9 La vicenda del premio, su cui non mi dilungo, e il contenuto della prima versione sono stati oggetto di una tesi di carattere storico redatta da J. Barrow Green [6]. 
paragrafo 13 del primo capitolo Poincaré formula quello che lui stesso definisce le problème général de la dynamique: Studiare la dinamica di un sistema canonico con Hamiltoniana

$$
H(p, q)=H_{0}(p)+H_{1}(p, q), \quad p \in \mathcal{G} \subset \mathbb{R}^{n}, q \in \mathbb{T}^{n},
$$

dove $\mathcal{G}$ è un aperto.

Il primo tentativo, tutto sommato naturale, consiste nel far ricorso a un teorema già dimostrato da Liouville, e che si enuncia brevemente come segue. Se l'Hamiltoniana $H(p, q)$ ammette un sistema di n integrali primi $\Phi_{1}(p, q), \ldots, \Phi_{n}(p, q)$ in involuzione allora il sistema delle equazioni di Hamilton è integrabile per quadrature. Qui le coordinate canoniche $p, q$ sono da intendersi in senso generico, non necessariamente nel senso di variabili d'azione e angolo come invece si suppone per l'Hamiltoniana (13). Il teorema di Liouville è stato riformulato da Arnold [4], affermando che se per una scelta $c_{1}, \ldots, c_{n}$ di costanti la varietà definita dalle equazioni $\Phi_{1}(p, q)=c_{1}, \ldots, \Phi_{n}(p, q)=c_{n}$ ammette una componente connessa $e$ compatta allora quella varietà è un toro $n$-dimensionale, $e$ in un suo intorno si possono introdurre variabili d'azione e angolo $p \in \mathcal{G} \subset \mathbb{R}^{n}, q \in$ $\mathbb{T}^{n}$ tali che l'Hamiltoniana in queste variabili dipende solo dalle azioni $p$. La dimostrazione è riportata in forma incompleta nel lavoro citato di Arnold, ed è stata poi completata da Jost [38] (si veda anche [37]).

Ai nostri giorni è d'uso chiamare integrabile tout court un sistema Hamiltoniano a cui si applica il teorema di Arnold-Jost. ${ }^{10}$ Il fatto che l'Hamiltoniana $H(p)$ dipenda solo dalle azioni $p$ ha conseguenze molto forti. Scrivendo le equazioni canoniche

$$
\dot{q}=\frac{\partial H}{\partial p}=: \omega(p), \quad \dot{p}=0
$$

si vede subito che la soluzione corrispondente al dato inizale $q_{0}, p_{0}$ si scrive

$$
q(t)=q_{0}+\omega\left(p_{0}\right) t, \quad p(t)=p_{0} .
$$

Dunque a ciascun dato iniziale corrisponde un flusso di Kronecker su un toro $\mathbb{T}^{n}$, con frequenze $\omega(p)$ dipendenti dal valore iniziale delle azioni.

10 Il lettore potrà legittimamente osservare che il problema dei due corpi, ad esempio, non potrebbe dirsi integrabile, perché il moto uniforme del baricentro non si descrive mediante un angolo. Ma si sottintende che moti di questo genere siano già stati eliminati mediante l'introduzione di coordinate opportune, come in effetti si fa per il problema dei due corpi $\mathrm{o}$, più in generale, per sistemi a più corpi o per il corpo rigido. 
Nelle coordinate originarie del problema (ad esempio le coordinate cartesiane o polari per il problema planetario) l'evoluzione diventa una serie di Fourier che rappresenta un moto quasi periodico, ossia una sovrapposizione di moti periodici con frequenze $\left\langle k, \omega\left(p_{0}\right)\right\rangle$ costruite combinando le $n$ frequenze fondamentali $\omega\left(p_{0}\right)$.

L'applicazione al Sistema Solare richiede un minimo di attenzione, perché un sistema di $n$ pianeti che si muovono nello spazio ha $3 n$ gradi di libertà (e dunque la dimensione $n$ che compare nella formula (13) dovrebbe riscriversi come $3 n$ ). Si dovrebbero dunque osservare $3 n$ frequenze fondamentali, mentre la descrizione kepleriana ne individua solo $n$, una per pianeta. Le $2 n$ frequenze mancanti nascono dalla teoria di Lagrange, in quanto $n$ di esse corrispondono alla precessione dei perieli, e altre $n$ alla precessione dei nodi. ${ }^{11}$ Il fatto notevole è però questo: se si potesse dimostrare che l'Hamiltoniana (13) ammette $n$ integrali primi si arriverebbe immediatamente alla conclusione che il Sistema Solare è stabile. Sarebbe, di fatto, la rappresentazione dei moti planetari mediante epicicli, e in conseguenza di ciò le frequenze osservate dovrebbero essere tutte combinazioni di $3 n$ frequenze fondamentali; cosa, quest'ultima, ignota agli astronomi greci. Va da sé che un tale risultato sarebbe in qualche senso equivalente a dimostrare che le serie puramente trigonometriche costruite con metodi perturbativi sono uniformemente convergenti per tutti i tempi, che è esattamente il problema proposto nel bando del premio del Re di Svezia.

Qui arriva il primo risultato negativo di Poincaré, che in qualche senso sancisce la non integrabilità del sistema (13). A tal fine egli considera lo sviluppo in serie di Fourier dell'Hamiltoniana, scrivendo

$$
H_{1}(p, q)=\sum_{k \in \mathbb{Z}^{n}} h_{k}(p) e^{i\langle k, q\rangle},
$$

con coefficienti funzioni (olomorfe) delle azioni $p$.

Teorema 1: Supponiamo che l'Hamiltoniana (13) soddisfi le ipotesi seguenti:

(i) non degenerazione, ossia

$$
\operatorname{det}\left(\frac{\partial^{2} H_{0}}{\partial p_{j} \partial p_{k}}\right) \neq 0
$$

11 In realtà le frequenze legate alla precessione dei nodi sono solo $n-1$, in conseguenza della conservazione del momento angolare. Ma ciò non è rilevante ai fini della discussione che segue, e non entro in ulteriori dettagli. 
(ii) genericità: nessuno dei coefficienti $h_{k}(p)$ dello sviluppo di Fourier di $H_{1}(p, q)$ si annulla identicamente sulla varietà $\langle k, \omega(p)\rangle=0$. Allora non esistono integrali primi uniformi indipendenti da $H$.

Il teorema appena enunciato dimostra di fatto che il problema generale della dinamica è genericamente non integrabile. ${ }^{12}$ Si potrebbe sollevare qualche obiezione sull'applicabilità al problema planetario. La più forte, immediata, è che l'Hamiltoniana (6) non soddisfa la condizione di non degenerazione, perché $H_{0}(\Lambda)$ dipende solo da $n$ azioni. Analogamente, il teorema non si applicherebbe all'Hamiltoniana secolare (12), perché nell'approssimazione quadratica si avrebbe una funzione lineare delle azioni. Queste difficoltà si superano ricorrendo a metodi di forma normale del resto già ampiamente applicati da Poincaré, anche se è d'uso parlare di forma normale di Birkhoff. Lo spazio di questa nota non mi consente di dilungarmi oltre su questo punto.

Resterebbe una via d'uscita: evitare l'uso di variabili d'azione e angolo e puntare direttamente alla dimostrazione della convergenza uniforme per tutti i tempi delle serie perturbative, in qualunque forma siano esse ottenute. In effetti, nel secondo volume di Méthodes Nouvelles Poincaré passa in rassegna i metodi perturbativi sviluppati fino a quel momento e dimostra che tutti producono serie divergenti, con una sola eccezione che resta dubbia: quella delle serie di Lindstedt. Ma anche per queste conclude che ritiene la convergenza del tutto inverosimile, pur in mancanza di un argomento risolutivo.

\subsection{L'intersezione omoclina e il caos}

Poincaré dedica una parte consistente di Méthodes Nouvelles allo studio delle orbite periodiche e delle soluzioni asintotiche ad esse associate. Queste ultime sono particolarmente interessanti proprio perché non presentano quelle caratteristiche di quasi periodicità che sono l'obiettivo tipico degli sviluppi perturbativi. Qui si presenta il fenomeno dei punti omoclini e l'insorgere di comportamenti caotici.

Lo strumento tecnico introdotto e ampiamente usato da Poincaré viene da lui chiamato théorie des conséquents. Oggigiorno si parla più comunemente di sezione di Poincaré. Per illustrarlo conviene prendere in

12 La dimostrazione si può trovare nel trattato di Poincaré [70]. Il teorema è stato generalizzato da Fermi [18]. Un'ulteriore generalizzazione si può trovare in [7]. 
considerazione il modello del pendolo forzato, descritto dall'equazione

$$
\ddot{x}+\sin x=\varepsilon \cos \nu t
$$

dove $\nu$ è la frequenza della forzante ed $\varepsilon$ è un parametro che deve pensarsi piccolo. Il modello non è molto lontano da quanto avviene nel Sistema Solare: la perturbazione di Giove sui pianeti interni o sugli asteroidi della fascia tra Marte e Giove può ben schematizzarsi mediante l'azione di una forzante periodica su un oscillatore non lineare.

$\operatorname{Per} \varepsilon=0$ si ha la consueta equazione del pendolo. Se ricorriamo alla ben nota rappresentazione sul piano di fase le soluzioni asintotiche di Poincaré in questo caso sono le separatrici che connettono gli equilibri instabili $x= \pm \pi$. Il movimento corrispondente, che nessuno ha mai realizzato con un pendolo vero, è un'orbita che tende asintoticamente all'equibrio superiore del pendolo sia per $t \rightarrow-\infty$ che per $t \rightarrow+\infty$, avendo fatto un solo giro. La domanda è: cosa accade quando si accende la perturbazione ponendo $\varepsilon \neq 0$ ?

Lo studio qualitativo viene sensibilmente facilitato se si ricorre alla sezione di Poincaré a periodo fissato. In altri termini, si ricorre a uno stroboscopio che illumina istantaneamente la scena allo scadere di ciascun periodo $T=2 \pi / \nu$ della forzante, e si marca sul piano di fase la posizione e la velocità del pendolo in quell'istante. A ogni condizione iniziale corrisponde un'orbita, ovvero una successione di punti, che rappresenta la successione temporale degli stati, quelli che Poincaré chiama les conséquents.

Al giorno d'oggi si fa una cosa molto semplice: si prova a integrare numericamente l'equazione e si vede cosa succede. Anzi, Chirikov nel 1969 ha proposto di sostituire l'integrazione col calcolo di una mappa, detta standard map, che imita il comportamento di un pendolo nel caso in cui la forzante sinusoidale venga sostituita da un piccolo impulso. Il vantaggio non banale è che il calcolo diventa molto più veloce - il che coi calcolatori di quegli anni non guastava. La mappa si scrive

$$
x^{\prime}=x+y(\bmod 2 \pi), \quad y^{\prime}=y-\varepsilon \sin (x+y)(\bmod 2 \pi),
$$

dove $(x, y) \in \mathbb{T}^{2}$, e ha la proprietà interessante di conservare l'area si tratta di una mappa canonica, o simplettica che dir si voglia. I punti $\left(-\frac{\pi}{2}, 0\right)$ e $\left(\frac{\pi}{2}, 0\right)$, che sul toro coincidono, corrispondono all'equilibrio superiore del pendolo. Da essi emanano le separatrici, la cui esistenza è garantita dal teorema della varietà stabile. 

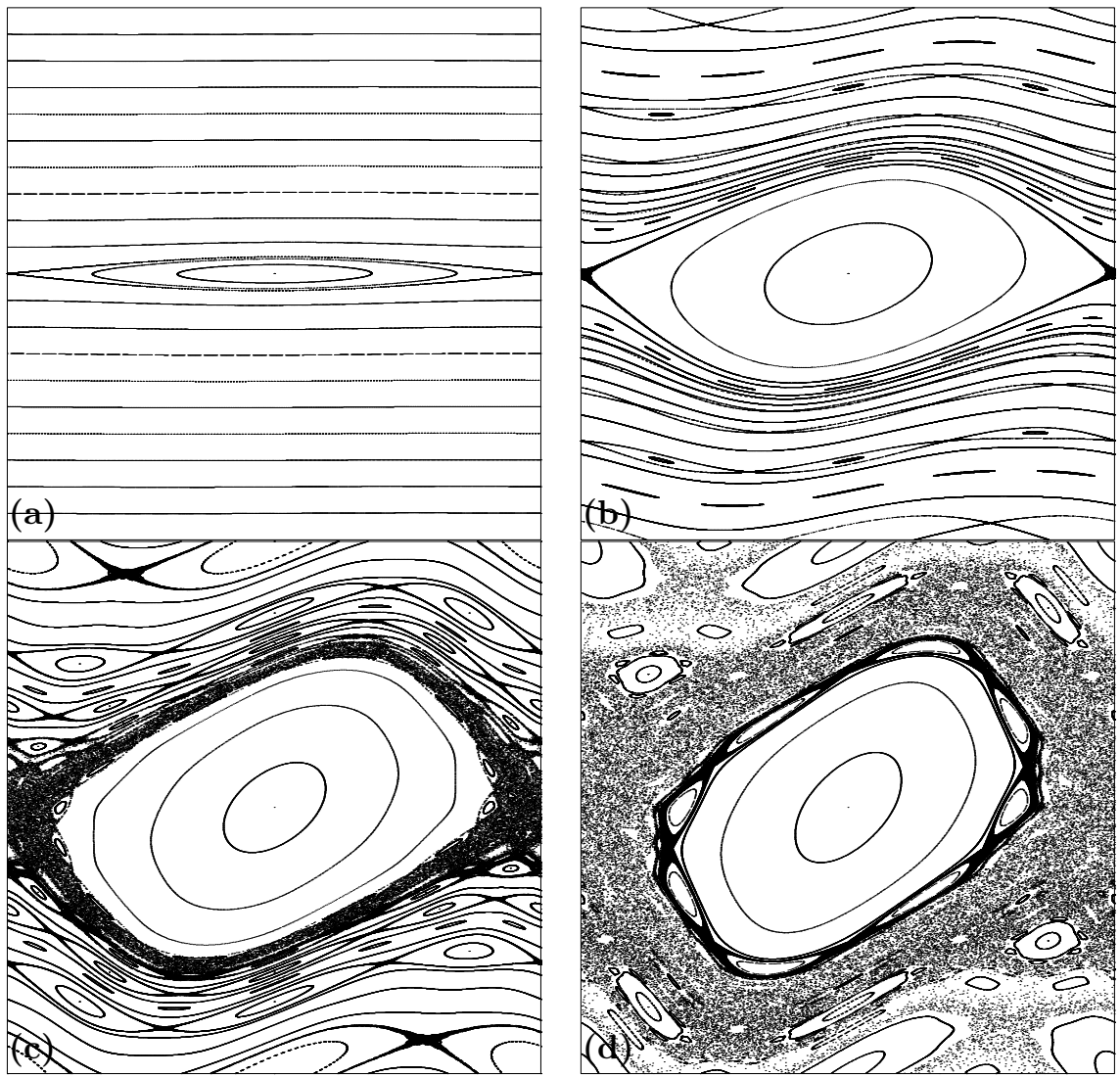

Fig. 4. La dinamica della standard map (14) per valori crescenti del parametro perturbativo $\varepsilon$. (a) $\varepsilon=0.001$; (b) $\varepsilon=0.4$, (c) $\varepsilon=0.8$, (d) $\varepsilon=1.1$.

La dinamica della mappa per valori crescenti di $\varepsilon$ è rappresentata in Fig.4. A prima vista il riquadro (a), che corrisponde a una perturbazione $\varepsilon$ molto piccola, non sembra differire molto dal diagramma di fase di un pendolo non forzato, ma nei riquadri successivi emerge con evidenza la coesistenza di zone di dinamica ordinata con altre dove si ha un comportamento imprevedibile. Il fenomeno è stato illustrato per la prima volta in un celeberrimo articolo di Hénon e Heiles [36], sorprendente proprio perché metteva in evidenza una dinamica caotica del tutto inaspettata in un modello estremamente semplice. In quell'articolo si usa l'immagine 
suggestiva delle "isole ordinate in un mare caotico", entrata poi nella letteratura. ${ }^{13}$ Un fatto da notare è che la zona caotica sembra nascere in prossimità dell'equilibrio instabile: lo si vede passando dal riquadro (b) al riquadro (c).

L'analisi di Poincaré si concentra sul comportamento delle varietà stabili e instabili. Per il pendolo imperturbato la varietà stabile e quella instabile del punto di equilibrio superiore coincidono e formano una coppia di separatrici. Ogni punto delle separatrici genera un'orbita doppiamente asintotica. Ma la domanda che Poincaré si pone è: cosa accade quando si accende la perturbazione? Il teorema della varietà stabile garantisce l'esistenza locale di un segmento della varietà nell'intorno del punto fisso, e l'esistenza globale si dimostra sfruttando la mappa stessa, unendo tutte le immagini iterate di quel segmento. Non c'è però nessuna garanzia che le varietà che coincidono per il sistema imperturbato continuino $\mathrm{a}$ coincidere anche sotto perturbazione.

L'errore di Poincaré nella prima versione della memoria per il premio del Re di Svezia sta proprio qui: egli ritiene che le varietà stabili e instabili continuino a coincidere. Lo riconosce lui stesso in una lettera a Mittag-Leffler, di cui riporto un brano.

"Mais les conséquences de cette erreur sont plus graves que je ne l'avais cru d'abord. Il n'est pas vrai que les surfaces asymptotiques soient fermées, au moins dans le sens où je l'entendais d'abord. Ce qui est vrai, c'est que si je considère les deux parties de cette surface (que je croyais hier encore raccordées l'une à l'autre) se coupent suivant une infinité de courbes trajectoires asymptotiques. J'avais cru que toutes ces courbes asymptotiques après sêtre eloignées d'une courbe fermée représentant une solution périodique, se rapprocheront ensuite asymptotiquement de la même courbe fermée, ce qui est vrai étant qu'il y en a une infinité qui jouisse de cette proprieté. Je ne vous dissimulerai pas le chagrin que me cause cette déconverte.

Il fenomeno è illustrato in Fig.5 facendo ricorso alla standard map con

13 Qualche anno prima, a far tempo dalla seconda metà degli anni '50, Contopoulos aveva studiato una classe di modelli simili a quello di Hénon e Heiles. Dalla lettura dei suoi lavori pubblicati intorno al 1960 emerge con evidenza che egli fosse a conoscenza del manifestarsi di una dinamica caotica. Questo, del resto, mi è stato confermato in un colloquio che ebbi anni fa con lui stesso. Ma egli diede molto peso alla costruzione di integrali primi approssimati che descrivevano bene i moti di tipo ordinato, e non pubblicò le figure che mostravano il caos. 

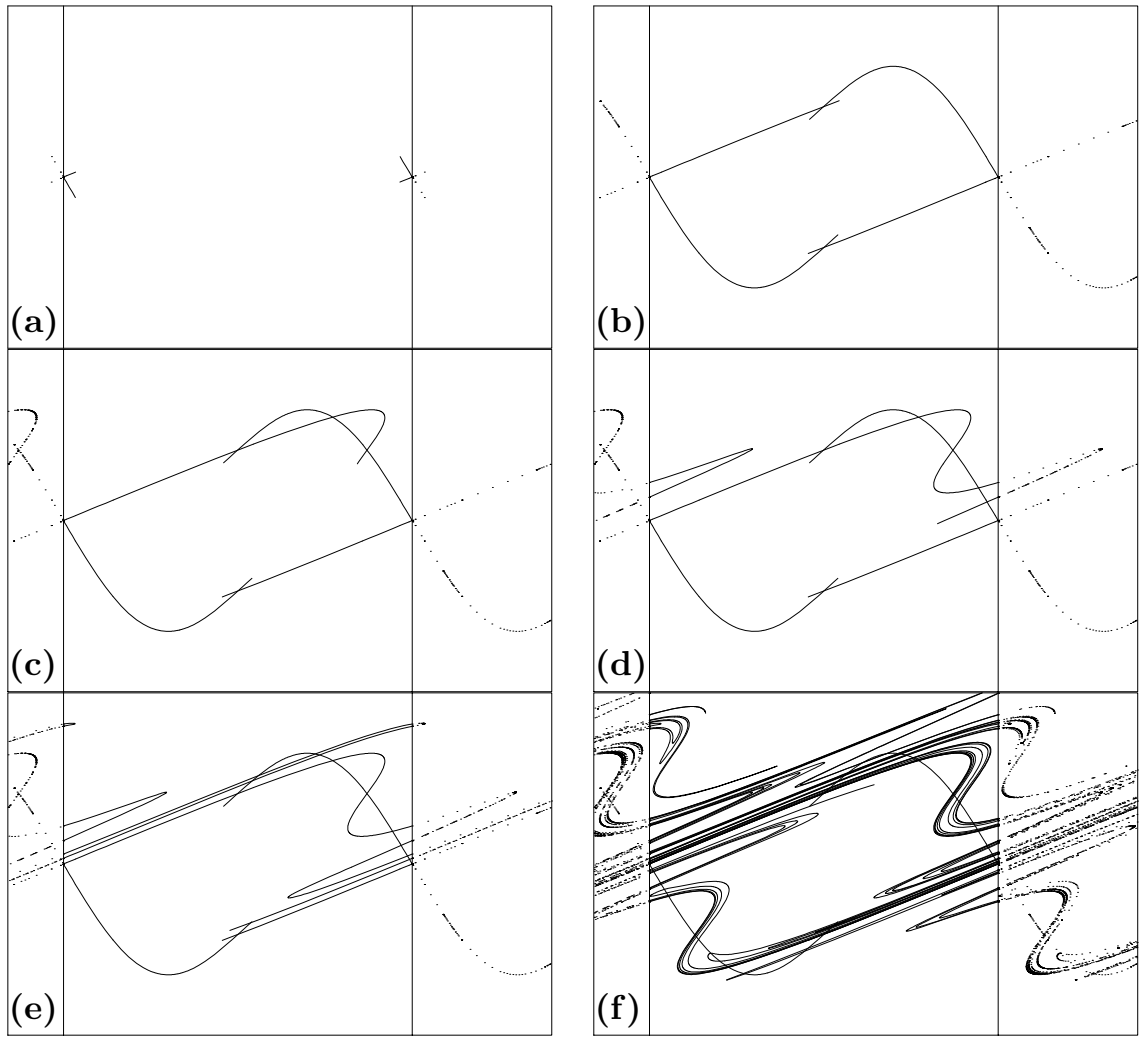

Fig. 5. Il fenomeno del punto omoclino per la standard map, con $\varepsilon=2.36$. Le due rette verticali delimitano il cilindro rappresentato sul piano, mentre le strisce aggiuntive con le curve punteggiate hanno lo scopo di facilitare la comprensione della figura. a. Il tratto iniziale delle varietà stabile ed instabile del punto fisso instabile, duplicato a causa della rappresentazione del cilindro sulpiano. b. Dopo 2 iterazioni le varietà si intersecano trasversalmente in due punti omoclini. c-f. Le immagini del primo tratto di varietà instabile del punto a sinistra dopo 3,4,5,8 iterazioni.

$\varepsilon=2.36$, un valore abbastanza alto al fine di rendere comprensibile la figura. Nel riquadro (a) ho rappresentato un piccolo tratto delle varietà stabili e instabili del punto fisso, che descrivono solo il comportamento locale. Il riquadro (b) rappresenta l'immagine dei tratti iniziali dopo 2 iterazioni: si vede che le due varietà si intersecano trasversalmente. Il punto 
di intersezione, chiamato da Poincaré punto omoclino, appartiene a entrambe le varietà, e quindi genera un'orbita doppiamente asintotica. Nei riquadri successivi, per non rendere la figura illeggibile, ho rappresentato solo le iterazioni successive del tratto di varietà instabile del punto a sinistra. Il fenomeno rilevante è la formazione di lobi dovuti alle intersezioni successive tra le due varietà; tutti i lobi hanno la stessa area, perché la mappa conserva l'area. Per inciso, già nel riquadro (c) si vede comparire un secondo punto omoclino, dovuto proprio alla conservazione dell'area. L'argomento di Poincaré, per cui rimando a Méthodes Nouvelles, è che di orbite omocline ne debbano esistere infinite, perché la varietà instabile non può intersecare se stessa, ma i lobi da essa formati devono diventare sempre più allungati, per via della conservazione dell'area, e reintersecare infinite volte la varietà stabile. Qui è d'obbligo lasciare la parola a Poincaré:

"Que l'on cherche à se représenter la figure formée par ces deux courbes et leurs intersections en nombre infini dont chacune correspond à une solution doublement asymptotique, ces intersections forment une sorte de treillis, de tissu, de reseau à mailles infiniment serrées; chacune de ces deux courbes ne doit jamais se recouper elle-même, mais elle doit se replier sur elle même d'une manière très complexe pour venir recouper une infinité de fois toutes les mailles du reseau.

On sera frappé de la complexité de cette figure, que je ne cherche même pas à tracer. Rien n'est plus propre à nous donner une idée de la complication du problème des trois corps et en général de tous les problèmes de Dynamique où il n'y a pas d'intègrale uniforme et où les séries de Boblin sont divergentes.

La disponibilità del calcolatore, combinata con una robusta dose di presunzione, mi induce a tentare la rappresentazione della figura che Poincaré si rifiuta di tracciare, limitandomi a una ventina di iterazioni dei segmenti iniziali di ambedue le varietà. Il risultato si vede in Fig.6.

Un fenomeno analogo può verificarsi anche nel caso di intersezione tra varietà stabili e instabili di punti fissi distinti: a tali intersezioni Poincaré dà il nome di punto eteroclino. Che ciò si verifichi risulta abbastanza evidente dalla Fig.4. Al crescere della perturbazione si osserva l'apparire di orbite periodiche dovute alle risonanze, con curve chiuse attorno alle orbite stabili e separatrici che emanano da quelle instabili. Allo stesso tempo la regione ove domina il comportamento caotico si espande fino a occupare una parte consistente dell'area disponibile. 


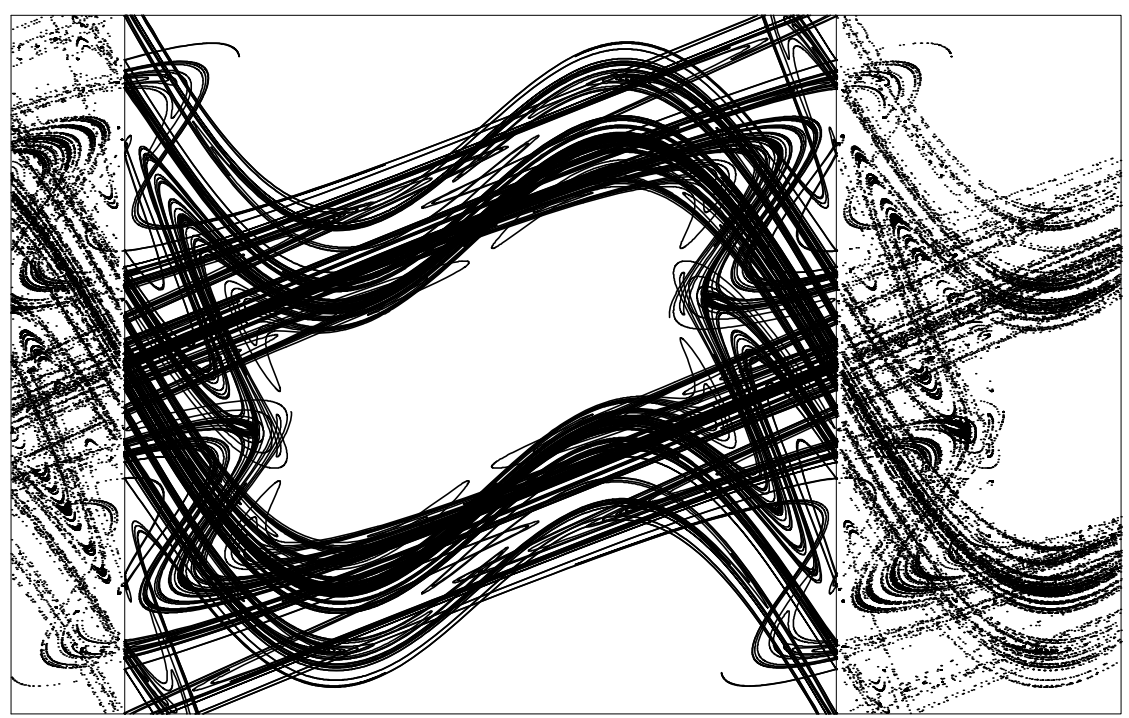

Fig. 6. Rappresentazione parziale delle varietà stabili e instabili per la standard map, $\operatorname{con} \varepsilon=2.36$.

Con la descrizione del fenomeno delle intersezioni omocline ed eterocline si chiude il terzo volume di Méthodes Nouvelles. Le ricerche sulla dinamica del Sistema Solare e in particolare sulla sua stabilità perdono quella centralità che avevano mantenuto per tutto il secolo XIX. Gli astronomi continuano a usare gli sviluppi classici, estremamente utili e produttivi nonostante che le serie siano divergenti; i fisici dirottano il loro interesse sulla Relatività e sulla Meccanica Quantistica; i matematici, anche grazie all'immenso lavoro di Poincaré, danno inizio a quella fase di sviluppo incredibile che ha caratterizzato il secolo XX fin dai suoi inizi. ${ }^{14}$

\section{LA QUiETE DOPO LA TEMPESTA}

A partire dagli anni '50 del secolo XX rinasce l'interesse sulla dinamica dei sistemi non lineari. Metterei l'attenzione su tre fatti: l'inizio

14 Tra le ricerche di interesse per il problema planetario meritano certamente di essere ricordate quelle di Levi-Civita [51][52][53] e Sundman [78] [79] [80] sulla regolarizzazione delle collisioni e sulla regolarità delle soluzioni del problema dei tre corpi. 
dell'esplorazione dello spazio; la disponibilità dei calcolatori elettronici; i primi risultati analitici sulla convergenza di serie con piccoli divisori.

Che l'esplorazione spaziale abbia dato nuovo impulso allo studio della dinamica planetaria è cosa che certo non sorprende. Non si tratta più di calcolare orbite di oggetti noti, o appena scoperti: per questo bastano i metodi tradizionali. Si tratta invece di calcolare traiettorie di satelliti artificiali, allo scopo di metterli in orbita intorno alla Terra o di inviarli sulla Luna, o ancora di inviarli a visitare pianeti, asteroidi e comete.

La disponibilità dei calcolatori elettronici viene presto sfruttata $\mathrm{da}$ Fermi che nel 1952 calcola la dinamica di una catena non lineare, ridiscutendo i fondamenti della Meccanica Statistica. Intorno al 1956 Contopoulos inizia a calcolare orbite di stelle in modelli galattici non lineari.

Sul fronte della matematica pura Siegel nel 1942 riesce per la prima volta a dimostrare la convergenza di serie perturbative in cui si presenta il problema dei piccoli divisori, nel caso di mappe del piano complesso [75]; nel 1952 estende i suoi risultati al caso di equazioni differenziali in un intorno di un equilibrio [76], superando così le difficoltà che avevano bloccato il lavoro di Poincaré. Nel 1954, al Congresso Internazionale dei Matematici di Amsterdam, Kolmogorov annuncia un teorema in cui si dimostra la persistenza di moti quasi periodici sotto perturbazione [43]. Quest'ultimo risultato sembra essere la conferma delle aspettative di Weierstrass che i moti planetari siano quasi periodici - si direbbe la rivincita definitiva degli epicicli. Ma la faccenda non è così semplice, e vorrei esporre con qualche dettaglio gli sviluppi successivi: qui si trova la giustificazione di quel "flebile sussurro" che ho messo nel titolo.

\subsection{Il teorema di Kolmogorov}

Per comprendere il teorema di Kolmogorov occorre tornare al problema generale della dinamica, ovvero l'Hamiltoniana (13), e soffermarsi un momento sul concetto di risonanza e sulla sua negazione, la non risonanza. Come ho già avuto modo di osservare, la dinamica dell'Hamiltoniana imperturbata $H_{0}(p)$ si descrive in modo semplice: lo spazio delle fasi è foliato in tori $\mathbb{T}^{n}$ invarianti parametrizzati dalle azioni $p$, e su ciascuno di questi tori si ha un flusso di Kronecker con frequenze $\omega(p)=\frac{\partial H_{0}}{\partial p}$ dipendenti dal toro. Le frequenze si dicono risonanti se vale una relazione $\langle k, \omega\rangle=0$ con $0 \neq k \in \mathbb{Z}^{n}$. La condizione di non degenerazione

$$
\operatorname{det}\left(\frac{\partial^{2} H_{0}}{\partial p_{j} \partial p_{k}}\right) \neq 0
$$


significa che le frequenze $\omega(p)$ possono prendersi come coordinate locali al posto delle azioni. Inoltre essa implica che sia i tori risonanti sia quelli non risonanti, ossia per cui $\langle k, \omega\rangle \neq 0$ per ogni $k \in \mathbb{Z}^{n}$ non nullo, sono densi nel dominio $\mathcal{G}$ delle azioni.

Era già noto a Poincaré che i tori risonanti vengono tipicamente distrutti dalla perturbazione. L'esempio più semplice è il caso di dimensione $n=2$. Un toro $\mathbb{T}^{2}$ risonante è a sua volta foliato in orbite periodiche. È spontaneo, tutto sommato, immaginare che una perturbazione, per quanto piccola, impedisca alle orbite di richiudersi in modo da restare periodiche, e infatti è ciò che tipicamente accade. Si riconosce questo fenomeno, con un po' di immaginazione, nella Fig.4. Nella sezione di Poincaré un'orbita periodica diventa un insieme finito di punti. Un toro risonante diventa una curva sulla quale tutti i punti sono periodici per la mappa. Le strutture formate da separatrici che racchiudono curve chiuse nascono proprio dalla perturbazione di tori risonanti: in generale sopravvive solo un numero finito e pari di orbite periodiche, metà stabili (circondate da curve), metà instabili (da cui emanano le separatrici). Si intuisce dunque che anche i tori vicini a quello risonante vengano distrutti.

Il fatto interessante riguarda proprio $i$ tori che sono in qualche senso lontani dalla risonanza. A tal fine è d'uso considerare frequenze che soddisfino la cosiddetta condizione diofantea

$$
|\langle k, \omega\rangle|>\frac{\gamma}{|k|^{\tau}}, \quad \gamma>0, \tau>n-1,
$$

dove $|k|=\left|k_{1}\right|+\ldots+\left|k_{n}\right|$. L'insieme delle frequenze che soddisfano tale condizione ha misura grande (proporzionale a $1-\gamma$ ), ma il suo complemento è aperto e denso. Dunque da un punto di vista probabilistico $\mathrm{i}$ tori fortemente non risonanti sono preponderanti, anche se il loro insieme sembra poco interessante dal punto di vista topologico. Il teorema di Kolmogorov afferma che i tori diofantei sopravvivono a una piccola perturbazione.

Teorema 2: Supponiamo che l'Hamiltoniana

$$
H(p, q)=H_{0}(p)+\varepsilon H_{1}(p, q), \quad p \in \mathcal{G} \subset \mathbb{R}^{n}, \quad q \in \mathbb{T}^{n}
$$

soddisfi l'ipotesi di non degenerazione

$$
\operatorname{det}\left(\frac{\partial^{2} H_{0}}{\partial p_{j} \partial p_{k}}\right) \neq 0 \text {. }
$$


Sia $p^{*}$ un toro invariante di $H_{0}(p)$ con frequenze $\omega^{*}=\omega\left(p^{*}\right)$ diofantee. Allora per $|\varepsilon|$ sufficientemente piccolo l'Hamiltoniana $H(p, q)$ ammette un toro invariante con frequenze $\omega^{*}$, prossimo al toro imperturbato $p^{*}$.

Può essere interessante richiamare i punti salienti della dimostrazione, sintetizzando la breve nota di Kolmogorov [43]. ${ }^{15}$ Alla base c'è l'osservazione che un'Hamiltoniana della forma (che chiamerò forma normale di Kolmogorov)

$$
H(p, q)=\langle\omega, p\rangle+\mathcal{R}(p, q), \quad \mathcal{R}(p, q)=O\left(p^{2}\right),
$$

composta da una parte lineare nelle azioni $p$ con frequenze costanti $\omega \in$ $\mathbb{R}^{n}$ e una parte non lineare $\mathcal{R}(p, q)$ che sia almeno quadratica nelle azioni, ammette evidentemente un toro invariante $p=0$. Infatti basta scrivere le equazioni di Hamilton corrispondenti,

$$
\dot{q}=\omega+O(p), \quad \dot{p}=O\left(p^{2}\right)
$$

e imporre le condizioni iniziali $q(0)=q_{0}, p(0)=0$, sicché il flusso è

$$
q(t)=q_{0}+\omega t, \quad p(t)=0,
$$

ossia un flusso di Kronecker con frequenze $\omega$ sul toro $p=0$. La domanda è se si possa ricondurre l'Hamiltoniana (16) alla forma (17). Il procedimento di Kolmogorov è il seguente. Si sviluppa l'Hamiltoniana in serie di Fourier degli angoli $q$ e in serie di Taylor delle azioni $p$ intorno al punto $p^{*}$ caratterizzato da frequenze imperturbate $\omega \in \mathbb{R}^{n}$ diofantee, ponendo così

15 Kolmogorov pubblicò solo una breve nota di sei pagine in cui diede una traccia della dimostrazione. Si potrebbe dire, in modo un po' irriverente: un esercizio di alto livello per studenti molto abili. Una decina d'anni dopo Arnold [2] [3] pubblicò una prova seguendo uno schema più generale (e decisamente più complicato) rispetto a quello di Kolmogorov. Nel frattempo Moser [63], venuto a conoscenza dell'enunciato di Kolmogorov ma ignorandone i dettagli, aveva pubblicato un risultato analogo, e di fatto equivalente, per le mappe di un anello che conservano l'area, rilassando anche la richiesta di analiticità e richiedendo solo che le funzioni avessero un numero sufficientemente alto di derivate. Questi lavori diedero inizio alla cosiddetta teoria $K A M$. Nella letteratura si trova spesso l'affermazione che Kolmogorov enunciò il teorema senza darne la dimostrazione. In realtà, secondo le testimonianze di contemporanei, egli tenne a Mosca una serie di lezioni in cui espose la prova completa, ma il testo non venne pubblicato in occidente. Per una dimostrazione che segue strettamente lo schema proposto da Kolmogorov nella sua breve nota si può vedere [8]. 
l'Hamiltoniana nella forma (traslando le azioni in modo che sia $p^{*}=0$ )

$$
\begin{aligned}
H(p, q)=\langle\omega, p\rangle+\frac{1}{2}\langle\mathrm{C} p, p\rangle & +A(q)+\langle B(q), p\rangle \\
+ & \frac{1}{2}\langle\mathrm{D}(q) p, p\rangle+O\left(p^{3}\right),
\end{aligned}
$$

con $A(q), B(q), \mathrm{D}(q)$ piccole, di ordine $\varepsilon$. Si cerca poi una trasformazione canonica prossima all'identità mediante una funzione generatrice

$$
S\left(p^{\prime}, q\right)=\left\langle p^{\prime}, q\right\rangle+X(q)+\langle\xi, q\rangle+\left\langle Y(q), p^{\prime}\right\rangle,
$$

dove il vettore $\xi \in \mathbb{R}^{n}$, la funzione $X(q)$ e la funzione vettoriale $Y(q)$ sono da determinarsi in modo da eliminare dall'Hamiltoniana i termini $A(q)$ e $\langle B(q), p\rangle$. Scrivendo la trasformazione canonica

$$
q^{\prime}=q+Y(q), \quad p=p^{\prime}+\frac{\partial X}{\partial q}+\xi+\left\langle\frac{\partial Y}{\partial q}, p^{\prime}\right\rangle
$$

si vede che essa consiste in una deformazione di coordinate periodica negli angoli $q$ alla quale si aggiunge una traslazione delle azioni $p$. Con un breve calcolo ci si rende conto che la piccola traslazione è necessaria per riassorbire la variazione delle frequenze indotta dalla trasformazione stessa, in modo che le frequenze $\omega$ restino fissate, e ciò è possibile grazie alla condizione di non degenerazione.

Applicando la trasformazione si ottiene una nuova Hamiltoniana che ha ancora la forma (18), ma con nuove funzioni, diciamo $A^{\prime}(q), B^{\prime}(q)$ che sono di ordine $\varepsilon^{2}$, o almeno così pare. La speranza è che iterando il procedimento infinite volte si possa costruire una successione di Hamiltoniane il cui limite

$$
H^{(\infty)}=\langle\omega, p\rangle+\mathcal{R}(p, q), \quad \mathcal{R}(p, q)=O\left(p^{2}\right),
$$

abbia la forma normale di Kolmogorov, e quindi ammetta un toro invariante che rispetto a quello imperturbato è deformato e traslato di poco.

Nella discussione della convergenza del procedimento si presenta il problema dei piccoli divisori. Per determinare $X(q)$ e $Y(q)$ occorre risolvere un'equazione della forma generale

$$
\left\langle\omega, \frac{\partial \chi}{\partial q}\right\rangle=\psi(q)
$$

dove $\psi(q)$ è una funzione nota e a media nulla e $\chi(q)$ è l'incognita. A tal fine si scrive lo sviluppo di Fourier $\psi(q)=\sum_{k \in \mathbb{Z}^{n}} c_{k} e^{i\langle k, q\rangle}$ con coefficienti 
$c_{k}$ noti, e con un breve calcolo si trova la soluzione nella forma

$$
\chi(q)=i \sum_{k \in \mathbb{Z}^{n}} \frac{c_{k}}{\langle k, \omega\rangle} e^{i\langle k, q\rangle} .
$$

La difficoltà nasce ancora una volta dalla presenza dei divisori $\langle k, \omega\rangle$, che non si annullano (grazie alla condizione di non risonanza), ma possono diventare arbitrariamente piccoli. Ora, se si assume che le frequenze siano diofantee e che la funzione $\psi(q)$ sia analitica si applica un argomento, già usato da Poincaré ([70], tomo II, cap. XIII), che prova l'analiticità della soluzione $\chi(q)$. L'argomento è il seguente. I coefficienti $c_{k}$ nello sviluppo di $\psi(q)$ soddisfano la diseguaglianza $\left|c_{k}\right|<C e^{-|k| \sigma}$, grazie all'analiticità di $\psi(q)$, con costanti $C$ e $\sigma$. La diseguaglianza diofantea $|\langle k, \omega\rangle|>\gamma /|k|^{\tau}$ significa che i coefficienti di $\chi(q)$ decadono come $|k|^{\tau} e^{-|k| \sigma} \sim e^{-|k| \sigma^{\prime}}$ con $\sigma^{\prime}<\sigma$. Dunque, la funzione generatrice per un singolo passo può determinarsi nonostante la presenza di piccoli divisori, ma serve una condizione di non risonanza forte. C'è però un prezzo: occorre rimpiazzare la costante $\sigma$ con una più piccola $\sigma^{\prime}$; in breve possiamo dire che c'è una perdita di analiticità. Il punto cruciale è che a ciascun passo del procedimento si ha una perdita simile, e non è evidente che l'accumulo di infinite perdite non distrugga la convergenza del procedimento. In effetti, questo è il punto su cui si era arrestato anche Poincaré.

Qui interviene Kolmogorov. Il suggerimento è di sfruttare l'osservazione fatta sopra che, almeno all'apparenza, una singola trasformazione canonica del tipo proposto da Kolmogorov riduce la taglia delle funzioni $A(q)$ e $B(q)$ da $\varepsilon$ a $\varepsilon^{2}$. Dunque, l'iterazione del procedimento condurrebbe passo passo a considerare termini di ordine $\varepsilon, \varepsilon^{2}, \varepsilon^{4}, \varepsilon^{8} \ldots$, una convergenza rapida che lo stesso Kolmogorov qualifica come "simile al metodo di Newton". Da qui le locuzioni "superconvergenza", "convergenza quadratica", "metodo quadratico" o simili, spesso associate in letteratura al teorema di Kolmogorov e considerate come un ingrediente essenziale della dimostrazione. ${ }^{16}$ La realtà è un po' più complicata.

16 Nota Rüssmann [72]: "It has often been said that the rapid convergence of the Newton iteration is necessary for compensating the influence of small divisors. But a deeper analysis shows that this is not true. (...) Historically, the Newton method was surely necessary to establish the main theorems of the KAM-theory. But for clarifying the structure of the small divisor problems the Newton method is not useful because it compensates not only the influence of small divisors, but also many bad estimates veiling the structure of the problems. "A mia conoscenza, la prima dimostrazione che non faccia uso del metodo quadratico è quella di Rüssman [73]. Per una 
L'accumulo dei divisori nel corso del procedimento viene compensato in modo sostanziale dalla decrescita rapida dovuta alle potenze di $\varepsilon$, e si può completare la dimostrazione mostrando, ad esempio, che la taglia delle funzioni da eliminare decresce al passo $r$-esimo come $1 / r^{2}$. Comunque convergente, sicché si arriva a dimostrare il teorema, ma tutta la potenza del metodo quadratico viene spesa per controllare i divisori.

L'interesse del teorema di Kolmogorov per il problema della dinamica planetaria è stato sottolineato fin dall'inizio, e presentato come la soluzione del problema della stabilità del Sistema Solare. Arnold [3] scrive:

"One of the most remarkable of A.N. Kolmogorov's mathematical achievements is his work on classical mechanics of 1954. A simple and novel idea, the combination of very classical and essentially modern methods, the solution of a 200 year-old problem, a clear geometrical picture and great breadth of outlook - these are the merits of the work."

Moser [64], riallacciandosi alla convinzione di Poincaré che le serie di Lindstedt siano divergenti, scrive:

"With the work of Kolmogorov and Arnold we know that, in fact, the opposite is the case and that these series expansions do converge and represent bona fide solutions of the problem, at least if a certain Hessian determinant does not vanish. Thus we can say that Weierstrass' question is finally answered in the positive sense. Moreover, this is not in contradiction to Poincaré's theorem on the nonexistence of integrals.

La difficoltà insita nell'ipotesi di non degenerazione è giustificata: come ho già avuto modo di osservare, l'Hamiltoniana imperturbata del sistema planetario è degenere. Tuttavia essa viene superata nel secondo lavoro di Arnold sul teorema di Kolmogorov [3].

Tutto sembra risolto, finalmente, ... ma resta ancora qualche dubbio. Il fatto è che l'enunciato del teorema dice che i tori invarianti esistono e hanno misura grande se la perturbazione $\varepsilon$ è abbastanza piccola. Ma è spontaneo chiedersi: quanto piccola? si tratta di una condizione soddisfatta per il Sistema Solare, o almeno per una parte di esso, ad esempio per i pianeti maggiori? ${ }^{17}$

dimostrazione che fa uso sostanzialmente degli sviluppi classici in un parametro si può vedere [24] [25] [26].

17 A questo proposito vorrei citare un aneddoto, riferito da Contopoulos ([16], $\S 7)$. Dopo una conferenza di Moser, che aveva presentato il teorema KAM, Con- 
Un secondo dubbio riguarda l'insieme dei dati iniziali a cui il teorema si applica. L'insieme dei tori invarianti, ha misura grande, ma il suo complemento è aperto e denso, e per $n>2$ è connesso. Un'orbita che ha dato iniziale nel complemento potrebbe benissimo visitarlo praticamente tutto. Questo fenomeno è stato descritto in un esempio da Arnold [5], ed è stato poi chiamato diffusione di Arnold. Ora, i dati iniziali dei pianeti sono noti solo entro un certo grado di approssimazione. Come decidere se corrispondono o no a un toro invariante? E come tener conto, oltre che dei pianeti, anche dei loro satelliti e almeno degli asteroidi più massicci?

\subsection{Il teorema di Nekhoroshev}

Una via d'uscita dalle questioni precedenti consiste nel rivedere il concetto di stabilità di un sistema. Le definizioni che si riallacciano alle ricerche di Lyapounov richiedono proprietà che siano valide per tutti $i$ tempi. Ma nessun sistema fisico, per quanto ne sappiamo, esiste per un tempo infinito. Un buon suggerimento è cercare risultati validi per tempo finito ma abbastanza lungo da coprire l'intervallo di vita del sistema stesso, chiedendo allo stesso tempo che valgano per dati iniziali in un aperto. Ad esempio, per il Sistema Solare si può richiedere un tempo dell'ordine di qualche miliardo di anni - l'età stimata del sistema stesso, o dell'universo. Per un acceleratore di particelle si può parlare di diversi giorni - la durata di un esperimento. Per una galassia si può osservare che le stelle compiono qualche centinaio di rivoluzioni in un tempo dell'ordine dell'età dell'universo - come dire che la galassia può ben esistere senza essere molto stabile: un vortice di polvere che presto si dissolve.

A mia conoscenza, il primo risultato è dovuto a Moser [62]. Pochi anni dopo Littlewood [57] [58] pubblica due lavori sulla stabilità dei punti triangolari di Lagrange per i problema dei tre corpi ristretto. Per una formulazione generale dobbiamo attendere i lavori di Nekhoroshev [65] [66].

topoulos osservò che nei grafici di sezioni di Poincaré calcolate numericamente si vedevano orbite che stavano su tori invarianti - fatte salve le incertezze dovute agli inevitabili errori numerici; domandò quindi se dalla dimostrazione si potesse avere un'idea di quanto dovesse essere piccola la perturbazione. Moser affermò che una tal stima non era disponibile. Hénon, che era presente, riuscì a darne una stima rozza servendosi delle diseguaglianze contenute nel lavoro di Moser, e il giorno dopo comunicò che risultava $\varepsilon \sim 10^{-48}$. Nel caso del sistema Sole-Giove-Saturno ciò significa assumere che la massa di Giove sia dell'ordine di quella del protone. 
Teorema 3: Supponiamo che l'Hamiltoniana $H=H_{0}(p)+\varepsilon H_{1}(p, q)$ sia analitica in un dominio $\mathcal{G} \times \mathbb{T}^{n}$, con $\mathcal{G}$ aperto, e che $H_{0}(p)$ sia convessa ${ }^{18}$ in $\mathcal{G}$. Allora esistono costanti positive $\varepsilon^{*} e T^{*}$ tali che se $\varepsilon<\varepsilon^{*}$ allora per ogni orbita $p(t), q(t)$ con dato iniziale $p(0) \in \mathcal{G}$ vale la diseguaglianza

$$
\operatorname{dist}(p(t)-p(0)) \leq \varepsilon^{1 / 4}
$$

per tutti i tempi t che soddisfano

$$
|t| \leq \frac{T^{*}}{\varepsilon} \exp \left[\left(\frac{\varepsilon^{*}}{\varepsilon}\right)^{1 / 2 a}\right], \quad a \sim n^{2}+n .
$$

L'interesse è duplice. Il primo punto notevole è la stima esponenziale del tempo di stabilità: al decrescere di $\varepsilon$ il tempo cresce così rapidamente da rendere concepibile la possibilità di ottenere risultati validi per l'età dell'universo. Con una felice espressione di Littlewood: "If not eternity, this is a considerable slice of it". Il secondo punto, altrettanto notevole, è che il risultato vale per dati iniziali in un aperto, il che aiuta a superare il problema dell'inevitabile imprecisione sui dati iniziali.

Si deve osservare che l'aperto in questione può ben contenere risonanze. L'esistenza di orbite caotiche e di fenomeni di diffusione non è esclusa: il teorema afferma solo che caos e diffusione possono indurre variazioni consistenti delle azioni del sistema, ed eventuali fenomeni di instabilità, solo su tempi estremamente lunghi. Alla luce di queste osservazioni il teorema di Nekhoroshev presenta un interesse notevole per le applicazioni a sistemi fisici. Resta però, anche qui, la domanda: quanto deve essere piccola la perturbazione?

Il significato del teorema si comprende meglio, probabilmente, se si dà una versione locale. A tal fine si considera un sistema canonico nell'intorno di un equilibrio ellittico, descritto dall'Hamiltoniana

$$
\begin{aligned}
& H(x, y)=H_{0}(x, y)+H_{1}(x, y)+\ldots, \\
& H_{0}(x, y)=\sum_{j} \frac{\omega_{j}}{2}\left(x_{j}^{2}+y_{j}^{2}\right), \quad(x, y) \in \mathbb{R}^{n},
\end{aligned}
$$

dove $H_{s}(x, y)$ è un polinomio omogeneo di grado $s+2$. Tale è, ad esempio, l'Hamiltoniana che descrive i moti secolari delle variabili lente, op-

18 Il lavoro originale di Nekhoroshev richiede che l'Hamiltoniana imperturbata $H_{0}(p)$ soddisfi una proprietà di steepness, più debole rispetto alla convessità. L'uso della convessità semplifica sensibilmente la dimostrazione, ed è stato introdotto nella prima prova pubblicata in occidente [9]. 
pure quella che descrive la dinamica nell'intorno dei punti triangolari di Lagrange per il problema dei tre corpi.

A tale sistema si può applicare la teoria della forma normale di Birkhoff. Si cerca una trasformazione canonica prossima all'identità che ponga il sistema nella forma ${ }^{19}$

$$
Z(x, y)=H_{0}(I)+Z_{1}(I)+\ldots+Z_{r}(I)+\mathcal{F}^{(r+1)}(x, y)
$$

con $Z_{1}, \ldots, Z_{r}$ funzioni solo delle azioni $I_{j}=\frac{x_{j}^{2}+y_{j}^{2}}{2}$ e con $\mathcal{F}^{(r+1)}(x, y) \mathrm{di}$ grado almeno $r+3$. Ciò è possibile se si assume una condizione di non risonanza sulle frequenze $\omega$.

Birkhoff non entra nella discussione della convergenza della forma normale, ma osserva che se si arresta il procedimento a un ordine $r$ finito allora l'Hamiltoniana (20) è certamente analitica in un intorno dell'origine. Si può allora porre la questione della stabilità nei termini seguenti. Dal momento che la parte quadratica $H_{0}$ dell'Hamiltoniana iniziale (19) ammette gli $n$ integrali primi indipendenti $I_{1}, \ldots, I_{n}$ (le azioni degli oscillatori) si può cercare di ottenere una diseguaglianza del tipo

$$
\left|I_{j}(t)-I_{j}(0)\right|<\delta,
$$

con $\delta$ piccolo, che sia valida su un intervallo di tempo sufficientemente lungo. Si può anche formulare una proprietà più debole, che seguirebbe dalla precedente. Si osserva che il polidisco di raggio $\varrho$ intorno all'origine

$$
\Delta_{\varrho}=\left\{(x, y): x_{j}^{2}+y_{j}^{2} \leq \varrho^{2} R_{j}^{2}\right\}
$$

è palesemente invariante per il flusso generato da $H_{0}$. Si punta a dimostrare che se il dato iniziale è contenuto in un polidisco $\Delta_{\varrho-\delta}($ ad esempio con $\delta=\varrho / 2$ ) allora l'orbita resta confinata in $\Delta_{\varrho}$ per un tempo molto lungo. ${ }^{20}$ Se ora consideriamo la forma normale $(20)$ troviamo che nel po-

19 La costruzione formale si puo trovare in innumerevoli testi o articoli, a partire dal trattato di Birkhoff [10]. Una dimostrazione della stima esponenziale si può trovare in [23].

20 Se le frequenze $\omega$ hanno tutte lo stesso segno allora l'invarianza di un polidisco $\Delta_{\varrho}$ sufficientemente piccolo segue direttamente dalla conservazione dell'energia, perché $H(x, y)$ ha un estremo nell'equilibrio; non serve aggiungere altro. Ciò è stato osservato da Dirichlet. Da qui non segue però la proprietà più forte espressa dalla (21). Il problema diventa decisamente più complicato se le frequenze hanno segni diversi, perché in tal caso l'argomento di Dirichlet non si applica. Un esempio notevole è quello dei punti triangolari di Lagrange del problema dei tre corpi. 
lidisco $\Delta_{\varrho}$ vale una stima del tipo

$$
\left|\mathcal{F}^{(r+1)}(x, y)\right| \leq B^{r} \varrho^{r+3},
$$

dove $B$ è una quantità dipendente da $r$ che Birkhoff non cerca di determinare. Osservando che le azioni $I$ della forma normale hanno derivata temporale $\dot{I}=\{H, I\}=\left\{\mathcal{F}^{(r)}, I\right\} \sim \varrho^{r+3}$ possiamo concludere che la diseguaglianza (21) voluta con $\delta=O\left(\varrho^{3}\right)$ vale fino a un tempo $T=$ $O\left(1 / \varrho^{r}\right)$. A questa proprietà Birkhoff ha dato il nome "stabilità completa".

Si può fare di meglio introducendo uno schema di stime quantitative, assumendo ancora una volta una condizione di non risonanza di tipo diofanteo tra le frequenze. Tali stime non consentono di dimostrare che per $r \rightarrow+\infty$ la forma normale è convergente - il che garantirebbe stabilità perpetua, alla Lyapounov. Per $r$ finito si ha però una stima del tipo

$$
\left|\mathcal{F}^{(r+1)}(x, y)\right| \leq(r !)^{n} B^{r} \varrho^{r+3}
$$

dove $B$ non dipende più da $r$. Dal momento che $r$ è arbitrario lo si può scegliere come funzione $r(\varrho)$ che minimizzi la stima a destra. Si trova così una stima esponenziale del tipo di Nekhoroshev

$$
r \sim \frac{1}{\varrho^{1 / n}}, \quad\left|\mathcal{F}^{\left(r_{\mathrm{opt}}\right)}(x, y)\right| \sim \exp \left(-(1 / \varrho)^{1 / n}\right) .
$$

Grazie a questa si migliora sensibilmente la teoria della stabilità completa di Birkhoff.

Teorema 4: Sia data l'Hamiltoniana (19), e supponiamo che le frequenze $\omega \in \mathbb{R}^{n}$ siano diofantee. Allora esistono due costanti positive $\varrho^{*}$ $e T^{*}$ tali che per $\varrho<\varrho *$ si ba

$$
|I(t)-I(0)|=O\left(\varrho^{3}\right)
$$

per un tempo $|t|<T_{\text {stab }}$ con

$$
T_{\text {stab }}=T^{*} \exp \left(\left(\varrho^{*} / \varrho\right)^{1 / n}\right) .
$$

Ne segue, ad esempio, che ogni orbita con dato iniziale in un polidisco $\Delta_{\varrho / 2}$ resta confinata nel polidisco $\Delta_{\varrho}$ almeno fino al tempo esponenzialmente lungo dato dal teorema.

Anche qui, la domanda interessante è se $\varrho^{*}$ possa assumere valori realistici per un sistema fisico. ${ }^{21}$

21 Ad esempio, l'applicazione di stime analitiche nel caso dei punti triangolari di La- 


\subsection{La stabilità superesponenziale}

Prima di chiudere questo paragrafo vorrei accennare brevemente a un risultato ancora più forte, dovuto a Morbidelli e all'autore, che prolunga ulteriormente e in modo significativo il tempo stimato di stabilità. Lo si può enunciare in due forme: in ambito locale o globale (in un senso da precisare).

In ambito locale si considera ancora un intorno $\Delta_{\varrho}$ di un equilibrio ellittico, con l'Hamiltoniana (19). Si costruisce ancora la forma normale di Birkhoff (20) e si raccoglie in un'unica funzione la parte normalizzata, integrabile. Si può dunque scrivere, in variabili d'azione e angolo,

$$
Z(x, y)=\mathcal{Z}_{0}(p)+\varepsilon \mathcal{Z}_{1}(p, q),
$$

identificando $\varepsilon \mathcal{Z}_{1}(p, q)=\mathcal{F}^{(r+1)}(x, y)$. Si ottiene così un'Hamiltoniana che ha la forma del problema generale della dinamica, ma il parametro $\varepsilon$ della perturbazione, grazie alla stima esponenziale (23), è valutabile come $\varepsilon \sim \exp \left(-(1 / \varrho)^{1 / n}\right)$. A questa Hamiltoniana, nel dominio $\Delta_{\varrho}$, si può applicare la formulazione generale del teorema 3 di Nekhoroshev, ottenendo così un tempo di stabilità del tipo

$$
T \sim \exp \left(\exp \left(1 / \varrho^{1 / n}\right)\right)
$$

Un argomento analogo si applica, con piccolissime varianti, all'intorno di un toro invariante, partendo da un'Hamiltoniana nella forma normale di Kolmogorov [61]. Va da sé che un tal risultato diventa alquanto significativo, perché pur non superando il problema della diffusione di Arnold dimostra comunque che tale diffusione è estremamente lenta, più che esponenzialmente. ${ }^{22}$ Torna alla mente il brano di Newton che ho riportato nel paragrafo 1 .

In modo più generale si può partire direttamente dall'Hamiltoniana $H(p, q)=H_{1}(p)+\varepsilon H_{1}(p, q)$ su un dominio $\mathcal{G} \times \mathbb{T}^{n}$. La formulazione diventa allora globale nel senso che non ci si restringe all'intorno di un equilibrio: il dominio $\mathcal{G}$ può ben coprire una parte consistente

grange del problema ristretto circolare dei tre corpi, nel caso del sistema Sole-Giove, ha dato come risultato $\varrho^{*} \sim 1 \mathrm{Km}$. Ciò è ben poco rispetto alla distanza tra Giove e il Sole, che è circa $8 \times 10^{8} \mathrm{Km}$; difficile dunque considerare un tal risultato come significativo per la stabilità degli asteroidi cosiddetti troiani [23]. Con le tecniche descritte nel paragrafo 5 le stime sono state migliorate fino a circa $1 / 6$ della distanza Sole-Giove [13][32].

22 Per un risultato più debole di stabilità esponenziale si può vedere [68]. 
dello spazio delle azioni [30]. Spiegare il procedimento qui è più difficile, perché richiede una qualche familiarità con lo schema della dimostrazione del teorema di Kolmogorov ideato da Arnold [3]. Mi limito quindi a descrivere in modo informale il risultato.

L'idea di base consiste nel troncare lo sviluppo di Fourier dell'Hamiltoniana $H_{1}(p, q)$ conservando solo un numero finito di termini, e procedere, ancora una volta, alla costruzione di una forma normale di Birkhoff a ordine finito. A prima vista un tal procedimento sembra accettabile per chi, come gli astronomi, intende svolgere dei calcoli al fine di determinare, ad esempio, le effemeridi dei pianeti, ma è più ostico per chi cerca risultati rigorosi nel senso che a questo aggettivo danno i matematici. Un problema, questo, a cui Poincaré era molto sensibile.

Il punto è che si può dare al procedimento una forma rigorosa seguendo la traccia dello schema di Arnold a cui ho accennato sopra. L'osservazione che sta alla base, del resto già messa in evidenza da Poincaré, è che grazie all'analiticità delle funzioni, e al conseguente decadimento esponenziale dei coefficienti dello sviluppo di Fourier, si può spezzare la perturbazione $\varepsilon H_{1}(p, q)$ in una parte che è effettivamente di ordine $\varepsilon$ (un polinomio trigonometrico, diciamo di grado $K$ ), una seconda parte di ordine $\varepsilon^{2}$, e poi $\varepsilon^{3}, \varepsilon^{4} \ldots$, che sono polinomi trigonometrici di grado $2 K, 3 K, 4 K \& \mathrm{cc}^{23}$ Il fatto che le frequenze $\omega(p)=\frac{\partial H_{1}}{\partial p}$ dipendano dalle azioni significa solo che una condizione di non risonanza, ad esempio di tipo diofanteo, deve essere soddisfatta in un sottodominio chiuso (ma con interno non vuoto) di $\mathcal{G}$, che comunque ha misura relativa grande. Applicando più volte una formulazione adattata del teorema di Nekhoroshev e partendo da un dominio $\mathcal{D}^{(0)}=\mathcal{G}^{(0)} \times \mathbb{T}^{n}$ si costruisce una successione di domini

$\mathcal{D}^{(0)}=\mathcal{G}^{(0)} \times \mathbb{T}^{n} \supset \mathcal{D}^{(1)}=\mathcal{G}^{(1)} \times \mathbb{T}^{n} \supset \ldots \supset \mathcal{D}^{(r)}=\mathcal{G}^{(r)} \times \mathbb{T}^{n} \supset \ldots$

all'interno di ciascuno dei quali l'Hamiltoniana ha la forma $H^{(r)}(p, q)=$ $H_{0}^{(r)}(p)+\varepsilon_{r} H_{1}^{(r)}(p, q)$, ma con $\varepsilon_{r} \sim \exp \left(-1 / \varepsilon_{r-1}\right)$. Se ne conclude che in ciascuno di questi domini vale una stima di stabilità fino a un tempo

$$
T_{r} \sim \underbrace{\exp \circ \ldots \circ \exp }_{r \text { volte }}(1 / \varepsilon) \text {. }
$$

23 Un ragionamento spontaneo induce a scegliere $K \sim-\ln \varepsilon$. In realtà tale scelta si rivela ingenua e inefficace: occorre di fatto scegliere $K$ abbastanza grande, ma indipendente da $\varepsilon$. Si veda ad esempio la nota 39 in [21]. 
Ancor più interessante, la successione dei domini converge a un insieme di tori invarianti che corrisponde in qualche modo a quello dei tori di Kolmogorov.

\section{IL TEMPO DEI SOGNI}

Nel paragrafo precedente, dopo gli enunciati dei teoremi di Kolmogorov e Nekhoroshev, ho sollevato il problema dell'applicabilità di questi teoremi al mondo fisico, e in particolare al Sistema Solare - o almeno a una parte di esso. Qui vorrei affrontare questo problema mostrando come almeno in qualche caso si possano ottenere risultati realistici.

Pensare di ottenere stime realistiche con metodi puramente analitici è un atteggiamento molto ottimistico, ma poco produttivo. La via d'uscita consiste nel ricorrere a metodi dimostrativi che fanno uso del calcolatore (spesso denominati “computer assisted proof'). In questo caso si fa ricorso a sviluppi perturbativi espliciti. Qui è d'obbligo premettere due considerazioni.

La prima è che occorre formulare degli algoritmi costruttivi, che richiedano la manipolazione di un numero finito di termini - tipicamente polinomi algebrici o trigonometrici. Fin qui, poco di diverso rispetto a quanto gli astronomi hanno fatto dai tempi di Lagrange in poi. Ma l'applicazione di teoremi quali quello di Kolmogorov o Nekhoroshev richiede anche valutazioni quantitative che nel caso di Kolmogorov devono arrivare fino alla convergenza, faccende che gli astronomi prendono assai raramente in considerazione - e ben a ragione.

La seconda considerazione è che gli algoritmi devono essere tradotti in programmi che facciano uso di manipolazione algebrica al calcolatore. Gli algoritmi espliciti sono applicabili al calcolo diretto, in linea di principio, e del resto matematici e astronomi del passato hanno spesso dedicato tempo ed energie considerevoli per svolgere tali calcoli manualmente. Ma le applicazioni di cui sto parlando richiedono la manipolazione di milioni di coefficienti: il ricorso al calcolatore è inevitabile. Le applicazioni di cui discuterò nel resto del paragrafo fanno uso di un pacchetto di manipolazione algebrica sviluppato dall'autore in collaborazione con U. Locatelli e M. Sansottera [31].

\subsection{Applicazioni del teorema di Kolmogorov}

Il lavoro preliminare consiste nel costruire un algoritmo esplicito per cal- 
colare la forma normale di Kolmogorov, facendo uso di sviluppi in un parametro perturbativo, secondo lo schema classico, con l'aggiunta di un riordinamento dell'Hamiltoniana che consenta di operare a ogni passo con un numero finito di termini negli sviluppi di Fourier. In questo schema l'algoritmo delle serie di Lie per generare trasformazioni canoniche si è rivelato molto utile. Il procedimento formale non è dissimile da quello descritto nel paragrafo 4.1, salvo il fatto che risulta più comodo fare uso di due funzioni generatrici

$$
\chi_{1}(q)=X(q)+\langle\xi, q\rangle, \quad \chi_{2}=\langle Y(q), p\rangle
$$

trasformando poi l'Hamiltoniana $H$ in

$$
H^{\prime}=\exp \left(L_{\chi_{2}}\right) \circ \exp \left(L_{\chi_{1}}\right) H,
$$

dove $L_{\chi} \cdot=\{\cdot, \chi\}$ è la derivata di Lie, di fatto la parentesi di Poisson con $\chi$. La differenza rispetto ai metodi abituali diventa sensibile nella dimostrazione della convergenza della successione infinita di trasformazioni, perché l'uso di un procedimento in stile classico non consente di applicare il metodo di convergenza quadratica. Occorre invece un'analisi abbastanza dettagliata del modo in cui i piccoli divisori si accumulano, verificando che lo fanno, per così dire, con gentilezza, senza compromettere la convergenza del procedimento [26]. Senza entrare in dettagli, il risultato che si trova è che il procedimento di costruzione della forma normale è convergente se le norme delle due successioni di generatrici $\chi_{1}, \chi_{2}$ che vengono costruite passo passo decadono a zero almeno geometricamente. Questa è la condizione che viene verificata mediante calcolo esplicito, almeno fino a un ordine finito.

Quella che vorrei descrivere sinteticamente è l'applicazione al problema dei tre corpi nel caso Sole-Giove-Saturno [59] [60]. Lo scopo è mostrare come si possa ottenere un risultato realistico. Occorrono diversi passi.

Il primo passo si riconduce alla teoria classica: occorre sviluppare l'Hamiltoniana del problema dei tre corpi in variabili di Poincaré, con i valori delle masse dei due pianeti maggiori. Il calcolo è laborioso, ma non presenta novità sostanziali rispetto a quanto gli astronomi hanno fatto per oltre un secolo, salvo che lo sviluppo viene spinto al secondo ordine nelle masse e si calcola un numero di termini trigonometrici molto più alto di quelli che si possono ottenere dal calcolo manuale.

Il secondo passo è l'eliminazione della degenerazione dell'Hamiltoniana imperturbata. A tal fine, seguendo lo schema ideato da Arnold [3], 

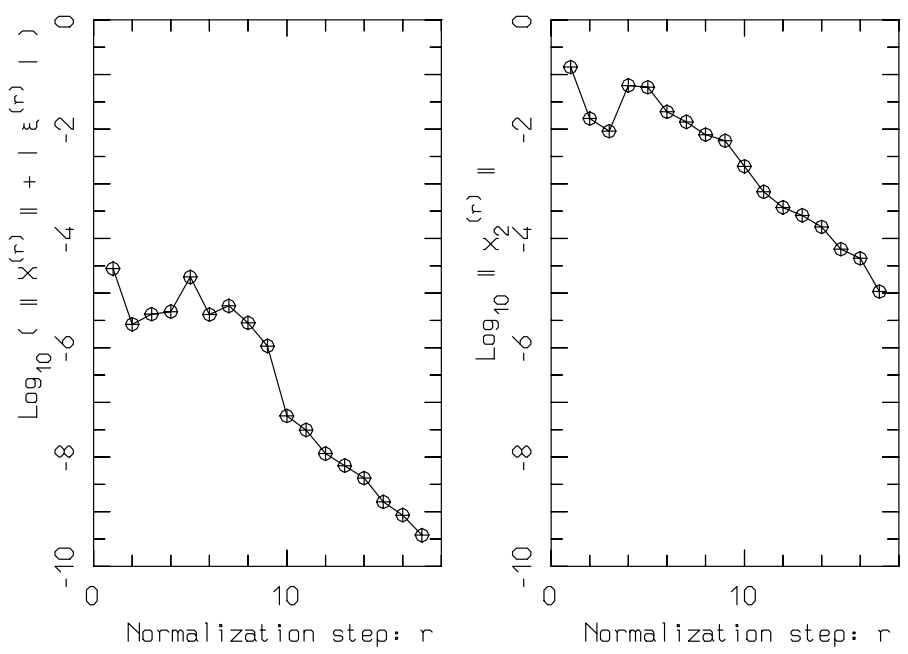

Fig. 7. Le norme delle generatrici della forma normale di Kolmogorov per i primi 17 passi di costruzione della forma normale, in funzione del passo. La scala verticale è logaritmica, sicché si vede che dopo qualche esitazione iniziale ambedue le norme presentano una decrescita geometrica regolare.

si calcola la forma normale di Birkhoff per le variabili lente, opportunamente troncata. Questa parte può considerarsi come un'estensione del calcolo di Lagrange che ho illustrato nel paragrafo 2.4: invece di fermarsi alla parte quadratica dell'Hamiltoniana, che sarebbe lineare nelle azioni e quindi degenere, si calcolano anche i termini non lineari fino al grado 6 , sicché l'Hamiltoniana imperturbata risulta essere non degenere e la perturbazione è di grado superiore al sesto nelle variabili polinomiali. Questa parte del calcolo risulta nuova rispetto ai procedimenti classici, ma è resa possibile proprio dal ricorso al calcolatore.

Il terzo passo consiste nel preparare l'Hamiltoniana per la costruzione della forma normale di Kolmogorov. A tal fine si reintroducono variabili d'azione e angolo anche per i moti lenti, e si determinano i valori delle azioni corrispondenti alle frequenze volute. Le frequenze dei moti lenti non sono determinabili direttamente dalle osservazioni, ma sono valutate mediante integrazione numerica delle equazioni su tempi sufficientemente lunghi.

Infine si applica il procedimento di Kolmogorov determinando così le funzioni generatrici $\chi_{1}^{(1)}, \chi_{2}^{(1)}, \chi_{1}^{(2)}, \chi_{2}^{(2)}, \ldots$ fino a un ordine finito (nel 
nostro caso 17). Avendo a disposizione le funzioni si calcolano le norme. Il risultato è illustrato in Fig.7, dove si vede chiaramente il decadimento geometrico dopo qualche incertezza nei primi passi.

Il risultato così ottenuto, se non è completamente rigoroso nel senso comunemente inteso in matematica, costituisce almeno un'indicazione molto forte dell'applicabilità del teorema al sistema Sole - Giove - Saturno: nell'approssimazione in cui si può trascurare l'azione di tutti gli altri pianeti, si può affermare che l'orbita di quel sistema è vicina a un toro invariante di Kolmogorov.

In letteratura si trovano altri lavori in cui si fa uso della manipolazione algebrica al fine di dimostrare l'esistenza di tori invarianti in sistemi di interesse fisico. Alcuni esempi riguardano l'accoppiamento spin-orbita [14], l'orbita dell'asteroide Veritas [15] e la dinamica in prossimità dei punti triangolari di Lagrange del problema ristretto dei tre corpi [19].

\subsection{Applicazioni del teorema di Nekhoroshev}

Mi limito qui a descrivere brevemente una delle applicazioni del teorema di Nekhoroshev al sistema Sole-Giove-Saturno [27][28], quella che si espone nel modo più rapido. In questo caso si procede a un'estensione della teoria di Lagrange per i moti secolari; occorrono diversi passi.

Il primo passo è analogo a quello necessario per l'applicazione del teorema di Kolmogorov: si sviluppa l'Hamiltoniana in variabili di Poincaré fino al secondo ordine nelle masse.

Il secondo passo consiste nell'introdurre il problema secolare come nella teoria di Lagrange, salvo l'aver tenuto conto del secondo ordine nelle masse. Grazie alla media sugli angoli veloci ci si riconduce a considerare solo eccentricità e inclinazioni, con gli angoli corrispondenti. Però, a differenza di Lagrange, non si fa uso della sola parte quadratica: si sviluppa invece l'Hamiltoniana in serie di potenze intorno all'orbita piana circolare, con eccentricità e inclinazioni nulle. $\mathrm{Ci}$ si riconduce così a un problema di perturbazione di un sistema di oscillatori armonici, al quale si applica il teorema 4.

Il terzo passo consiste nel costruire esplicitamente la forma normale di Birkhoff fino a un ordine abbastanza elevato, nel nostro caso 18, determinando anche a ogni ordine il resto non normalizzato denotato con $\mathcal{F}^{(r+1)}$ nella formula (20). In tal modo si hanno a disposizione tutte le approssimazioni della forma normale fino all'ordine 18 , con i relativi resti.

Qui interviene la scelta dell'ordine $r$ di normalizzazione. Seguendo 

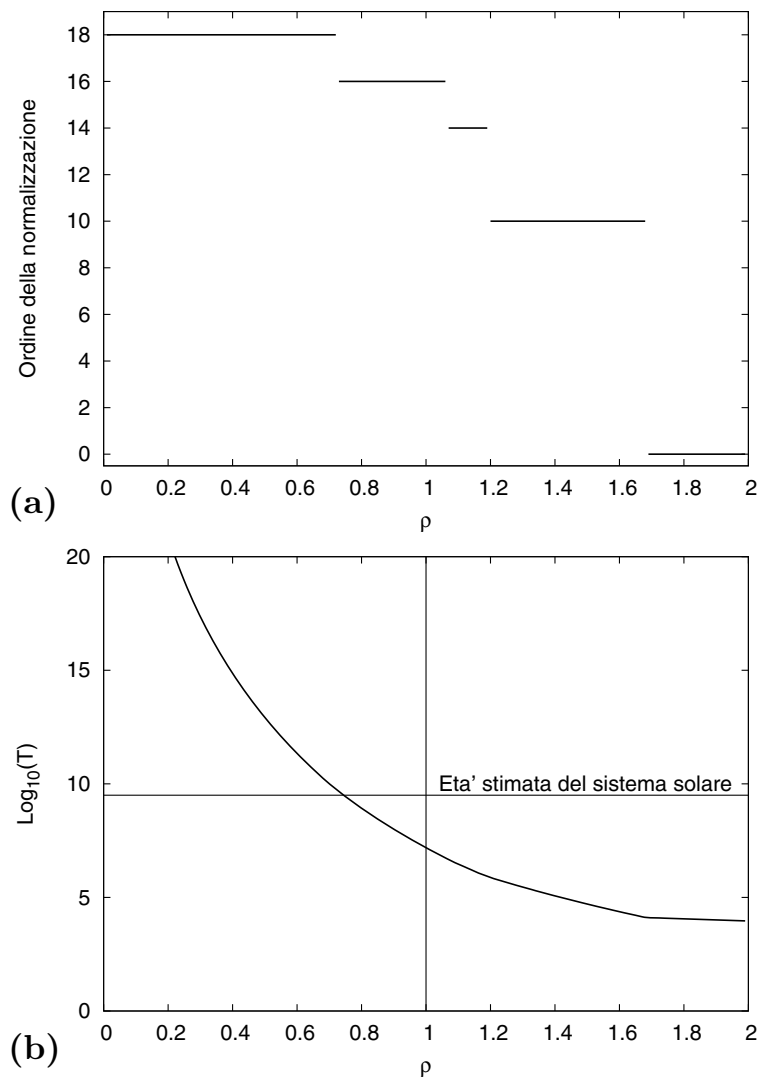

Fig. 8. (a) L'ordine ottimale di normalizzazione $r_{\max }$ in funzione di $\varrho$. (b) Stima del tempo di stabilità per il sistema Sole-Giove-Saturno al variare del raggio $\varrho$ del dominio contenente $i$ dati iniziali. I raggi $R$ per il calcolo della norma delle funzioni sono scelti in modo che $i$ dati reali per $i$ due pianeti corrispondano a $\varrho=1$, e l'unità di tempo è l'anno terrestre. La scala verticale riporta il logaritmo decimale del tempo. La linea tratteggiata orizzontale indica l'età stimata del sistema solare.

le indicazioni del paragrafo 4.2 si ricava l'ordine ottimale di troncamento in funzione del raggio $\varrho$ dell'intorno $\Delta_{\varrho}$. A tal fine per un $\varrho$ fissato si calcola la taglia del resto, e si sceglie il valore di $r$ che lo minimizza, trovando così una funzione a gradini di $\varrho$. La stima del tempo di stabilità come funzione di $\varrho$ si ricava usando quel valore di $r$. 
I risultati sono riassunti in Fig. 8 . Si vede che al diminuire di $\varrho$ il raggio ottimale cresce fino a 18 , che è l'ordine massimo della forma normale di Birkhoff. La crescita ovviamente continuerebbe se si calcolassero ordini più elevati. Il tempo di stabilità stimato cresce molto rapidamente al decrescere di $\varrho$, che rappresenta in pratica il valore opportunamente riscalato di eccentricità e inclinazione dei due pianeti. L'unità di misura è scelta in modo che le coordinate osservate dei pianeti si trovino al bordo di un dominio di raggio $\varrho=1$. Il grafico dà un tempo di circa $10^{7}$ anni, ancora al di sotto dell'età del Sistema Solare. Si vede tuttavia che tale età viene raggiunta già per $\varrho \sim 0.7$, e che viene moltiplicata per oltre $10^{4}$ per $\varrho \sim 0.6$. La stima dunque non è del tutto soddisfacente, ma occorre tener conto che la valutazione del resto richiede una stima del del massimo di un polinomio di grado elevato (fino a 19) su un polidisco. La crescita estremamente rapida del tempo stimato indica che una valutazione più accurata del resto potrebbe migliorare il risultato fino a raggiungere effettivamente l'età del Sistema Solare.

Vorrei sottolineare ancora una volta che la stabilità stimata con i metodi alla Nekhoroshev non esclude un comportamento caotico: dice solo che se il caos è presente riguarda una scala spaziale estremamente piccola e tempi estremamente lunghi.

In letteratura si possono trovare altre applicazioni della teoria di Nekhoroshev a sistemi reali. Come esempi posso citare il caso degli asteroidi troiani nell'intorno del punto triangolare di Lagrange $L_{4}$ del sistema Sole-Giove [23][13][32][77], la stabilità delle orbite nell'intorno di un toro invariante prossimo alle orbite del sistema formato da Sole, Giove, Saturno e Urano [29] e l'applicazione a sistemi extrasolari [55].

Gli esempi che ho riportato qui non danno una risposta definitiva al problema della stabilità, ma mettono in evidenza la possibilità che $\mathrm{i}$ tempi necessari perché il Sistema Solare si modifichi in modo sostanziale fino ad avere, ad esempio, collisioni tra i pianeti o espulsione di qualche pianeta, siano molto più lunghi della sua vita. Un sogno, questo, ma forse non del tutto irragionevole.

\section{Il FLebile SUSSURRo Del CAOS}

Non si può chiudere questa nota senza dedicare un breve spazio anche alle simulazioni numeriche. La teoria che ho illustrato nei paragrafi precedenti lascia immaginare che dare una descrizione completa in termini ana- 
litici della dinamica del Sistema Solare sia impresa a dir poco ardua, se non impossibile. D’altra parte non possiamo affidarci più di tanto alle osservazioni: anche tenendo conto di quelle più antiche si copre un intervallo temporale di meno di tremila anni, un'inezia rispetto alla vita del sistema che vorremmo studiare.

A questa mancanza si può sopperire ricorrendo alla simulazione numerica. Naturalmente occorre una potenza di calcolo considerevole se si vogliono calcolare le orbite planetarie su un arco di tempo paragonabile all'età stimata dell'Universo.

Le prime simulazioni su tempi lunghi sono state condotte a partire dalla fine degli anni '80 del secolo ormai trascorso da alcuni ricercatori tra cui A. Milani, M. Carpino, A. Nobili [12], G.J. Sussman, J. Wisdom [81], J. Laskar [49] [50]. Le loro conclusioni si possono riassumere così: i quattro pianeti maggiori (Giove, Saturno, Urano e Nettuno) sembrano muoversi in modo del tutto regolare anche su un arco di tempo di qualche miliardo di anni, che è l'età stimata del Sistema Solare. I pianeti interni (Mercurio, Venere, Terra e Marte) presentano invece delle piccole variazioni casuali delle orbite, in particolare dell'eccentricità, che non sono interpretabili come movimenti periodici: dobbiamo ammettere che c'è una componente caotica. Non che le orbite cambino di molto, almeno su tempi non troppo lunghi. Ma ci possono essere, ad esempio, piccole variazioni dell'eccentricità dell'orbita della Terra che hanno effetti molto rilevanti sul clima: le glaciazioni sembrano essere correlate proprio a queste variazioni.

Ancor più complessa è la situazione per gli asteroidi (che conosciamo da circa due secoli), e per gli oggetti transnettuniani (scoperta abbastanza recente). Qui l'azione delle risonanze e del caos che queste possono generare ha avuto tutto il tempo di dispiegarsi, e ne vediamo gli effetti nella disuniformità della distribuzione attuale di questi corpi. In questo contesto la presenza di una dinamica caotica diventa rilevante.

Il quadro che emerge è quello di un sistema in cui moti ordinati e caos sono mescolati in modo inestricabile. La dinamica dei pianeti e di molti asteroidi è ben approssimabile con moti quasi periodici, esattamente come gli astronomi hanno voluto fare per secoli. Ma a questi moti, perfettamente ordinati, si sovrappongono piccole variazioni imprevedibili che possono diventare visibili solo sull'arco di milioni o centinaia di milioni di anni. Un flebile sussurro, appunto, che però su un arco temporale di miliardi di anni può produrre effetti considerevoli, ad esempio lo stato del nostro sistema planetario quale lo osserviamo oggi. 


\section{BIBLIOGRAFIA}

[1] Alfonso X Rey de Castilla: Divi Alphonsi Romanorum et Hispaniarum regis, Astronomicae tabulae in propriam integritatem restitutae ..., Parisiis : ex officina Christiani Wecheli sub scuto Basiliensi, in vico Iacobaeo (1545).

[2] V.I. Arnold: Proof of a theorem of A.N. Kolmogorov on the invariance of quasiperiodic motions under small perturbations of the Hamiltonian, Usp. Mat. Nauk, 18, 13 (1963); Russ. Math. Surv., 18, 9 (1963).

[3] V.I. Arnold: Small denominators and problems of stability of motion in classical and celestial mechanics, Usp. Math. Nauk 18 N.6, 91 (1963); Russ. Math. Surv. 18 N.6, 85 (1963).

[4] V.I. Arnold: A theorem of Liouville concerning integrable problems of dynamics, Sibirsk. Math. Zh. 4, 471-474 (1963).

[5] V.I. Arnold: Instability of dynamical systems with several degrees of freedom, Sov. Math. Dokl. 5 N.1, 581-585 (1964).

[6] J. Barrow-Green: Poincaré and the three body problem, American Methematical Society (1997).

[7] G. Benettin, G. Ferrari, L. Galgani, A. Giorgilli: An extension of the PoincaréFermi theorem on the nonexistence of invariant manifolds in nearly integrable Hamiltonian systems, Nuovo Cimento B 72, 137-148 (1982).

[8] G. Benettin, L. Galgani, A. Giorgilli, J.M. Strelcyn: A proof of Kolmogorov's theorem on invariant tori using canonical transformations defined by the Lie method. Il Nuovo Cimento, 79, 201 (1984).

[9] G. Benettin, L. Galgani, A. Giorgilli: A proof of Nekhoroshev's theorem for the stability times in nearly integrable Hamiltonian systems. Cel. Mech., 37, 1-25 (1985).

[10] G.D. Birkhoff: Dynamical systems, New York (1927).

[11] M. Born: The mechanics of the atom, Frederick Ungar Publ. Co., New York (1927).

[12] M. Carpino, A. Milani, A. Nobili: Long term numerical integration and synthetic theories for the motion of outer planets, Astronomy and Astrophysics 181, 182-194 (1987).

[13] A. Celletti, A. Giorgilli: On the stability of the Lagrangian points in the spatial restricted problem of three bodies, Cel. Mech. 50, 31-58 (1991).

[14] A. Celletti: Construction of librational invariant tori in the spin-orbit problem, J. of Applied Math. and Physics (ZAMP), 45, 61-80 (1994).

[15] A. Celletti and L. Chierchia: KAM stability and Celestial Mechanics, Memoirs of AMS, 187, n. 878 (2007).

[16] G. Contopoulos: Adventures in order and chaos. A scientific autobiography, Kluver Academic Publishers (2004).

[17] C. Delaunay: Théorie du mouvement de la lune, Mem. 28 Acad. Sci. France, Paris (1860).

[18] E. Fermi: Generalizzazione del teorema di Poincaré sopra la non esistenza di integrali uniformi di un sistema di equazioni canoniche normali, Nuovo Cimento 26, 105-115 (1923). 
[19] F. Gabern, A. Jorba and U. Locatelli: On the construction of the Kolmogorov normal form for the Trojan asteroids, Nonlinearity, 18, n.4, 1705-1734 (2005).

[20] A. Giorgilli: A Kepler's note on secular inequalities, Rendiconti dell'Istituto Lombardo Accademia di Scienze e Lettere, Classe di Scienze Matematiche e Naturali, 145, 97-119 (2011).

[21] A. Giorgilli: Notes on exponential stability of Hamiltonian systems, in Dynamical Systems, Part I: Hamiltonian systems and Celestial Mechanics, Pubblicazioni del Centro di Ricerca Matematica Ennio De Giorgi, Pisa, 87-198 (2003).

[22] A. Giorgilli: I moti quasi periodici e la stabilità del sistema solare. I: Dagli epicicli al punto omoclino di Poincaré, Boll. Un. Mat. It. Sez. A, 10, 55-83 (2007).

[23] A. Giorgilli A. Delshams, E. Fontich, L. Galgani, C. Simó: Effective stability for a Hamiltonian system near an elliptic equilibrium point, with an application to the restricted three body problem. J. Diff. Eqs., 20, (1989).

[24] A. Giorgilli, U. Locatelli: Kolmogorov theorem and classical perturbation theory, ZAMP 48, 220-261 (1997).

[25] A. Giorgilli, U. Locatelli: On classical series expansions for quasi-periodic motions, MPEJ 3 N. 5 (1997).

[26] A. Giorgilli, U. Locatelli: A classical self-contained proof of Kolmogorov's theorem on invariant tori, in Proceedings of the NATO ASI school "Hamiltonian systems with three or more degrees of freedom”, C. Simó ed., NATO ASI series C: Math. Phys. Sci., Vol. 533, Kluwer Academic Publishers, Dordrecht-Boston-London, 72-89 (1999).

[27] A. Giorgilli, U. Locatelli, M. Sansottera: Kolmogorov and Nekhoroshev theory for the problem of three bodies, Cel. Mech and Dyn. Astr., 104 159-175 (2009).

[28] A. Giorgilli, U. Locatelli, M. Sansottera: Su un'estensione della teoria di Lagrange per $i$ moti secolari, Rendiconti dell'Istituto Lombardo Accademia di Scienze e Lettere, Classe di Scienze Matematiche e Naturali, 143, 223-239 (2010).

[29] A. Giorgilli, U. Locatelli, M. Sansottera: Secular dynamics of a planar model of the Sun-Jupiter-Saturn-Uranus system; effective stability into the light of Kolmogorov and Nekhoroshev theories, Reg. Ch. Dyn. 22, 54-77 (2017).

[30] A. Giorgilli, A. Morbidelli: Invariant KAM tori and global stability for Hamiltonian systems, ZAMP 48, 102-134 (1997).

[31] A. Giorgilli, M. Sansottera: Methods of algebraic manipulation in perturbation theory, in Chaos, Diffusion and Non-integrability in Hamiltonian Systems - Applications to Astronomy, Proceedings of the 3rd La Plata International School on Astronomy and Geophysics, P.M. Cincotta, C.M. Giordano and C. Efthymiopoulos eds., Universidad Nacional de La Plata and Asociación Argentina de Astronomía Publishers, La Plata, Argentina (2012).

[32] A. Giorgilli, Ch. Skokos: On the stability of the Trojan asteroids, Astron. Astroph. 317, 254-261 (1997).

[33] H. Gyldén: Untersuchungen über die Convergenz der Reigen, welche zur darstellung der Coordinaten der Planeten angewendet werden, Acta 9, 185-294 (1887).

[34] S.C. Haretu: Thèses presentès a la Faculté des Sciences de Paris, Gauthier-Villars, Paris, 1878. 
[35] S.C. Haretu: Sur l'invariabilité des grands axes des orbites planétaires, Ann. Obs. Paris, Mémoires, 18, 1-39 (1885).

[36] M. Hénon, C. Heiles: The applicability of the third integral of motion: some numerical experiments, Astron. J. 69, 73-79 (1964).

[37] H. Hofer, E. Zehnder: Symplectic invariants and Hamiltonian dynamics, Birkhäuser Verlag, Basel (1994).

[38] R. Jost: Winkel-und Wirkungsvariable für allgemeine mechanische Systeme, Helvetica Physica Acta 41, 965-968 (1968).

[39] J. Keplero: Astronomia Nova, seu Physica Coelestis tradita commentariis de motibus Stellæ Martis ex observationibus G.V. Tychonis Brahe; Jussu \& sumptibus Rudolphi II, Romanorum Imperatoris \&c. Plurium annorum pertinaci studio elaborata Pragx, A.S.C.M.S. Mathematico Johanne Keplero, cum ejusdem C.M. privilegio speciali, Anno æræ Dionysianæ MDCIX. Ristampato in: Johannis Kepleri astronomi opera omnia, edidit Dr. CH. Frisch, Frankfurti A.M. et Erlangæ Heyder \& Zimmer, MDCCCLX, Vol. III.

[40] J. Keplero: Tabulae Rudolphinae, quibus astronomicae scientiae, temporum longinquitate collapsae restauratio continentur. A Phonice illo Astronomorum Tychone ... primum animo concepta et destinata Anno Christi MDLXIV. Ulm: Jonas Saur, (1627).

[41] J. Keplero: In tabulas Rudolphi prafatio, incluso in: Johannis Kepleri astronomi opera omnia, edidit Dr. CH. Frisch, Frankfurti A.M. et Erlangx Heyder \& Zimmer, MDCCCLX, Vol. VI, pp 666-674.

[42] J. Keplero: Consideratio observationum Regiomontani et Waltheri, Incluso in: Johannis Kepleri astronomi opera omnia, edidit Dr. CH. Frisch, Frankfurti A.M. et Erlangæ Heyder \& Zimmer, MDCCCLX, Vol. VI, pp 725-774.

[43] Kolmogorov, A.N.: Preservation of conditionally periodic movements with small change in the Hamilton function, Dokl. Akad. Nauk SSSR, 98, 527 (1954). English translation in: Los Alamos Scientific Laboratory translation LA-TR-71-67; reprinted in: G. Casati, J. Ford: Stochastic behavior in classical and quantum Hamiltonian systems, Lecture Notes in Physics 93, 51-56 (1979).

[44] J.L. Lagrange: Solution de différents problèmes de calcul intégral, Miscellanea Tauriniensia, t.III (1762-1765). Ristampato in: Oeuvres de Lagrange, GauthierVillars, Paris (1867), tome I, pp 471-678

[45] J.L. Lagrange: 1776, Sur l'altération des moyens mouvements des planètes, Nouveaux Mémoires de l'Académie Royale des Sciences et Belles-Lettres de Berlin (1776). Ristampato in Oeuvres de Lagrange, Gauthier-Villars, Paris (1867), tome IV, pp 255-271.

[46] J.L. Lagrange: Théorie des variations séculaires del éléments des planètes. Première partie contenant les principes et les formules générales pour déterminer ces variations, Nouveaux mémoires de l'Académie des Sciences et Belles-Lettres de Berlin (1781). Ristampato in: Oeuvres de Lagrange, Gauthier-Villars, Paris (1870), tome V, p.125-207.

[47] J.L. Lagrange: Théorie des variations séculaires del éléments des planètes. Seconde partie contenant la détermination de ces variations pour chacune des planètes prin- 
cipales, Nouveaux mémoires de l'Académie des Sciences et Belles-Lettres de Berlin (1782). Ristampato in: Oeuvres de Lagrange, Gauthier-Villars, Paris (1870), tome V, p.211-489.

[48] P-S. de Laplace: Mémoire sur le principe de la gravitation universelle et sur les inégalités séculaires des planètes qui en dependent, Mémoires de l'Académie Royale des Sciences de Paris (1773). Ristampato in: Oeuvres complètes de Laplace, Gauthier-Villars, Paris (1891), tome VIII, p.201-275.

[49] J. Laskar: A numerical experiment on the chaotic behaviour of the solar system, Nature, 338, 237-238 (1989).

[50] J. Laskar: Large scale chaos in the solar system, Astron. Astroph. 287 (1994).

[51] T. Levi-Civita: Traiettorie singolari ed urti nel problema ristretto dei tre corpi, Annali di Matematica pura e applicata, serie III, IX (1904).

[52] T. Levi-Civita: Sur la résolution qualitative du problème restreint des trois corps, Acta Mathematica 30, 305-327 (1906).

[53] T. Levi-Civita: Sur la régularisation du problème des trois corps, Acta Mathematica 42 99-144 (1918).

[54] T. Levi-Civita, U. Amaldi: Lezioni di Meccanica Razionale, Zanichelli editore, Bologna (1927).

[55] A.-S. Libert and M. Sansottera: On the extension of the Laplace-Lagrange secular theory to order two in the masses for extrasolar systems, Celest. Mech. Dyn. Astr., 117, 149-168 (2013).

[56] A. Lindstedt: Beitrag zur integration der differentialgleichungen der differentialgleichungen der störungstheorie, Mém. Acad. Imp. des sciences St. Pétersbourg, XXXI N.4 (1883).

[57] J.E. Littlewood: On the equilateral configuration in the restricted problem of three bodies, Proc. London Math. Soc.(3) 9, 343-372 (1959).

[58] J.E. Littlewood: The Lagrange configuration in celestial mechanics, Proc. London Math. Soc.(3) 9, 525-543 (1959).

[59] U. Locatelli, A. Giorgilli: Invariant tori in the secular motions of the three-body planetary systems, Celestial Mechanics and Dynamical Astronomy, 78, 47-74 (2000).

[60] U. Locatelli, A. Giorgilli: Invariant tori in the Sun-Jupiter-Saturn system, DCDSB 7, 377 - 398 (2007).

[61] A. Morbidelli, A. Giorgilli: Superexponential stability of KAM tori, J. Stat. Phys. 78, 1607-1617 (1995).

[62] J. Moser: Stabilitätsverhalten kanonisher differentialgleichungssysteme, Nachr. Akad. Wiss. Göttingen, Math. Phys. Kl IIa, nr.6 (1955), 87-120.

[63] J. Moser: On invariant curves of area-preserving mappings of an annulus, Nachr. Akad. Wiss. Gött., II Math. Phys. Kl. 1962, 1-20 (1962).

[64] J. Moser: Stable and random motions in dynamical systems, Princeton University press, Princeton (1973).

[65] N.N. Nekhoroshev: Exponential estimates of the stability time of near-integrable Hamiltonian systems. Russ. Math. Surveys, 32, 1 (1977). 
[66] N.N. Nekhoroshev: Exponential estimates of the stability time of near-integrable Hamiltonian systems, 2. Trudy Sem. Petrovs., 5, 5 (1979).

[67] I. Newton: Philosophic Naturalis Principia Mathematica, Trin. Coll. Cantab. Soc. Matheseos Professore Lucasiano, \& Societatis Regalis Sodali, Londini, jussu Societatis Regix ac typis Josephi Streater, Anno MDCLXXXVII.

[68] A.D. Perry, S. Wiggins: KAM tori are very sticky: rigorous lower bounds on the time to move away from an invariant lagrangian torus with linear flow, Phys. D 71, 102-121 (1994).

[69] H. Poincaré: Sur le problème des trois corps et les équations de la dynamique, Acta Mathematica (1890).

[70] H. Poincaré: Les méthodes nouvelles de la mécanique céleste, Gauthier-Villars, Paris (1892).

[71] H. Poincaré: Leçons de Mécanique Céleste professées a la Sorbonne, Tome I, Théorie générale des perturbations planetaires, Gautier-Villars, Paris (1905).

[72] H. Rüssmann: Non-degeneracy in the perturbation theory of integrable dynamical systems, in Number theory and dynamical systems, Dodson, M.M., and Vickers, J.A.G., Eds., Cambr. Univ. Press (1989).

[73] H. Rüssmann: On the frequencies of quasi periodic solutions of analytic nearly integrable Hamiltonian systems, in Seminar on Dynamical Systems, S. Kuksin, V. Lazutkin and J. Pöschel eds., PNDLE 12, 160-183, Birkaüser Verlag (1994).

[74] M. Sansottera, U. Locatelli, A. Giorgilli: On the stability of the secular evolution of the planar Sun-Jupiter-Saturn-Uranus system, Mathematics and Computers in Simulation, Volume 88, 1-14 (2013).

[75] C.L. Siegel: Iteration of analytic functions, Annals of Math. 43, 607-612 (1942).

[76] C.L. Siegel: Über die Normalform analytischer Differentialgleichungen in der Nähe einer Gleichgewichtslösung, Nachr. Akad. Wiss. Göttingen, Math.-Phys. Kl. Math.-Phys.-Chem. Abt., 21-30 (1952).

[77] Ch. Skokos and A. Dokoumetzidis: Effective stability of the Trojan asteroids, Astron. Astroph., 367, 729-736 (2001).

[78] K.F. Sundman: Recherches sur le problème des trois corps, Acta Societatis Scientiarum FennicÆ XXXIV N. 6, (1906).

[79] K.F. Sundman: Nouvelles recherches sur le probleme des trois corps, Acta Societatis Scientiarum FennicÆ XXXV N. 9, (1909).

[80] K.F. Sundman: Mémoire sur le problème des trois corps, Acta Mathematica 36 105-179 (1912).

[81] G.J. Sussman, J. Wisdom: Numerical evidence that the motion of Pluto is chaotic, Science, 241, 433-437 (1988). 